RIMS-1718

Optimal matching forests and valuated delta-matroids

By

Kenjiro TAKAZAWA

$\underline{\text { March } 2011}$

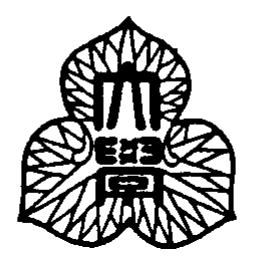

京都大学 数理解析研究所

RESEARCH INSTITUTE FOR MATHEMATICAL SCIENCES

KYOTO UNIVERSITY, Kyoto, Japan 


\title{
Optimal matching forests and valuated delta-matroids
}

\author{
Kenjiro Takazawa \\ Research Institute for Mathematical Sciences, \\ Kyoto University, Kyoto 606-8502, Japan. \\ takazawa@kurims.kyoto-u.ac.jp
}

March 16, 2011

\begin{abstract}
The matching forest problem in mixed graphs is a common generalization of the matching problem in undirected graphs and the branching problem in directed graphs. Giles presented an $\mathrm{O}\left(n^{2} m\right)$-time algorithm for finding a maximum-weight matching forest, where $n$ is the number of vertices and $m$ is that of edges, and a linear system describing the matching forest polytope. Later, Schrijver proved total dual integrality of the linear system.

In the present paper, we reveal another nice property of matching forests: the degree sequences of the matching forests in any mixed graph form a delta-matroid and the weighted matching forests induce a valuated delta-matroid. We remark that the delta-matroid is not necessarily even, and the valuated delta-matroid induced by weighted matching forests slightly generalizes the well-known notion of Dress and Wenzel's valuated delta-matroids. By focusing on the delta-matroid structure and reviewing Giles' algorithm, we design a simpler $\mathrm{O}\left(n^{2} m\right)$ time algorithm for the weighted matching forest problem. We also present a faster $\mathrm{O}\left(n^{3}\right)$-time algorithm by using Gabow's method for the weighted matching problem.
\end{abstract}

\section{Introduction}

The concept of matching forests in mixed graphs was introduced by Giles $[14,15,16]$ as a common generalization of matchings in undirected graphs and branchings in directed graphs. Let $G=$ $(V, E, A)$ be a mixed graph with vertex set $V$, undirected edge set $E$ and directed edge set $A$. Let $n$ and $m$ denote $|V|$ and $|E \cup A|$, respectively. For a vector $x \in \mathbf{R}^{E \cup A}$ and $F \subseteq E \cup A$, let $x(F):=\sum_{e \in F} x(e)$.

We denote a directed edge $a \in A$ from $u \in V$ to $v \in V$ by $u v$. A directed edge is often called an arc. For an arc $a=u v$, the terminal vertex $v$ is called the head of $a$ and denoted by $\partial^{-} a$, and the initial vertex $u$ is called the tail of $a$ and denoted by $\partial^{+} a$. For a vertex $v \in V$, the set of arcs whose head (resp., tail) is $v$ is denoted by $\delta^{-} v$ (resp., $\delta^{+} v$ ). For $B \subseteq A$, let $\partial^{-} B=\bigcup_{a \in B} \partial^{-} a$. A vertex in $\partial^{-} B$ is said to be covered by $B$. An arc subset $B \subseteq A$ is a branching if the underlying edge set of $B$ is a forest and each vertex $v \in V$ is the head of at most one $\operatorname{arc}$ in $B$. For a branching $B$, a vertex not covered by $B$ is called a root of $B$, and the set of the roots of $B$ is denoted by $R(B)$, i.e., $R(B)=V \backslash \partial^{-} B$.

An undirected edge $e \in E$ connecting $u, v \in V$ is denoted by $(u, v)$. We often abbreviate $(u, v)$ as $u v$, where it obvious that it is undirected. For $e=u v \in E$, both $u$ and $v$ are called as the head of $e$, and the set of heads of $e$ is denoted by $\partial e$, i.e., $\partial e=\{u, v\}$. For a vertex $v$, the set of edges incident to $v$ is denoted by $\delta v$. For $F \subseteq E$, let $\partial F=\bigcup_{e \in F} \partial e$. A vertex in $\partial F$ is said to be covered 
by $F$. An undirected edge subset $M \subseteq E$ is a matching if each vertex $v \in V$ is the head of at most one edge in $M$. A vertex not covered by $M$ is called a root of $M$ and the set of the roots of $M$ is denoted by $R(M)$, i.e., $R(M)=V \backslash \partial M$.

An edge set $F \subseteq E \cup A$ is a matching forest if the underlying edge set of $F$ is a forest and each vertex in $V$ is the head of at most one edge in $F$. Equivalently, an edge set $F=B \cup M$, where $B \subseteq A$ and $M \subseteq E$, is a matching forest if $B$ is a branching and $M$ is a matching with $\partial M \subseteq R(B)$. A vertex in $\partial^{-} B \cup \partial M$ are said to be covered by $F$, and a vertex is a root of $F$ if it is not covered by $F$. The set of the roots of $F$ is denoted by $R(F)$. Observe that $R(F)=R(B) \cap R(M)$ and $V=R(B) \cup R(M)$.

\section{$1.1 \quad$ Background}

Matching forests inherit the tractability of branchings and matchings. Let $w \in \mathbf{R}^{E \cup A}$ be a weight vector on the edge set of a mixed graph $G=(V, E, A)$. We consider the weighted matching forest problem, the objective of which is to find a matching forest $F$ maximizing $w(F)$. For this problem, Giles [15] designed a primal-dual algorithm running in $\mathrm{O}\left(n^{2} m\right)$ time, which provided a constructive proof for integrality of a linear system describing the matching forest polytope. Later, Schrijver [21] proved that Giles' linear system is totally dual integral. These results commonly extend the polynomial-time solvability and the total dual integrality results for the weighted branchings and weighted matchings $[4,7,9]$.

Topics related to matching forests include the following. Using the notion of matching forests, Keijsper [17] gave a common extension of Vizing's theorem [23, 24] on covering undirected graphs by matchings and Frank's theorem [11] on covering directed graphs by branchings. Another aspect of matching forests is that they can be represented as linear matroid matching (see [22]). From this viewpoint, however, we do not fully understand the tractability of matching forests, since the weighted linear matroid matching problem is unsolved while the unweighted problem is solved [18].

In the present paper, we reveal a relation between matching forests and delta-matroids $[1,3,5]$ to offer a new perspective on weighted matching forests which explains their tractability. For a finite set $V$ and $\mathcal{F} \subseteq 2^{V}$, the pair $(V, \mathcal{F})$ is a delta-matroid if it satisfies the following exchange property:

(DM) $\forall S_{1}, S_{2} \in \mathcal{F}, \forall s \in S_{1} \triangle S_{2}, \exists t \in S_{1} \triangle S_{2}, S_{1} \triangle\{s, t\} \in \mathcal{F}$.

Here, $\triangle$ denotes the symmetric difference, i.e., $S_{1} \triangle S_{2}=\left(S_{1} \backslash S_{2}\right) \cup\left(S_{2} \backslash S_{1}\right)$.

A typical example of a delta-matroid is a matching delta-matroid. For an undirected graph $G=$ $(V, E)$, let $\mathcal{F}_{M}=\{\partial M \mid M$ is a matching in $G\}$. Then, $\left(V, \mathcal{F}_{M}\right)$ is a delta-matroid [2,3]. Branchings in a directed graph also induce a delta-matroid, which we call a branching delta-matroid. For a directed graph $G=(V, A)$, let $\mathcal{F}_{B}=\{R(B) \mid B$ is a branching in $G\}$. Then, it is not difficult to verify that $\mathcal{F}_{B}$ is a delta-matroid (see $\S 2.1$ ).

A delta-matroid $(V, \mathcal{F})$ is called even if $\left|S_{1}\right|-\left|S_{2}\right|$ is even for any $S_{1}, S_{2} \in \mathcal{F}$. Note that a matching delta-matroid is an even delta-matroid, whereas a branching delta-matroid is not. Even delta-matroids are characterized by the following simultaneous exchange property [25]:

(EDM) $\forall S_{1}, S_{2} \in \mathcal{F}, \forall s \in S_{1} \triangle S_{2}, \exists t \in\left(S_{1} \triangle S_{2}\right) \backslash\{s\}, S_{1} \triangle\{s, t\} \in \mathcal{F}$ and $S_{2} \triangle\{s, t\} \in \mathcal{F}$.

The concept of valuated delta-matroids $[6,26]$ is a quantitative generalization of even deltamatroids. A function $f: 2^{V} \rightarrow \mathbf{R} \cup\{-\infty\}$ is a valuated delta-matroid if $\operatorname{dom} f \neq \emptyset$ and

$(\mathbf{V}-\mathbf{E D M}) \forall S_{1}, S_{2} \in \operatorname{dom} f, \forall s \in S_{1} \triangle S_{2}, \exists t \in\left(S_{1} \triangle S_{2}\right) \backslash\{s\}, f\left(S_{1} \triangle\{s, t\}\right)+f\left(S_{2} \triangle\{s, t\}\right) \geq$ $f\left(S_{1}\right)+f\left(S_{2}\right)$. 
Here, $\operatorname{dom} f:=\{S \mid S \subseteq V, f(S) \neq-\infty\}$. Note that $(V, \operatorname{dom} f)$ is an even-delta matroid. We remark here that weighted matchings in a weighted undirected graph induce a valuated deltamatroid $f_{M}$ with $\operatorname{dom} f_{M}=\mathcal{F}_{M}($ see $\S 2.1)$.

\subsection{Contributions}

In this paper, we consider delta-matroids commonly extending matching delta-matroids and branching delta-matroids, and also a valuation on those delta-matroids. For this purpose, we introduce a new class of delta-matroids which properly includes even delta-matroids. We call $(V, \mathcal{F})$ a simultaneous delta-matroid if it satisfies the following weaker simultaneous exchange property:

(SDM) $\forall S_{1}, S_{2} \in \mathcal{F}, \forall s \in S_{1} \triangle S_{2}, \exists t \in S_{1} \triangle S_{2}, S_{1} \triangle\{s, t\} \in \mathcal{F}$ and $S_{2} \triangle\{s, t\} \in \mathcal{F}$.

Note that every even delta-matroid is a simultaneous delta-matroid. Also, a branching matroid is a simultaneous delta-matroid (see $\S 2.1$ ).

The first main result in this paper is that matching forests also induce a simultaneous deltamatroid. For a mixed graph $G=(V, E, A)$, let $\mathcal{F}_{M F}=\{R(F) \mid F$ is a matching forest $\}$. We prove that $\mathcal{F}_{M F}$ is a simultaneous delta-matroid.

Theorem 1. For any mixed graph $G=(V, E, A)$, it holds that $\left(V, \mathcal{F}_{M F}\right)$ is a simultaneous deltamatroid.

Furthermore, we generalize the notion of valuated delta-matroids in order to deal with a quantitative extension of Theorem 1. That is, we define valuated delta-matroids on simultaneous deltamatroids, which slightly generalize valuated delta-matroids on even delta-matroids [6]. We call a function $f: 2^{V} \rightarrow \mathbf{R} \cup\{-\infty\}$ a valuated delta-matroid if $\operatorname{dom} f \neq \emptyset$ and

(V-SDM) $\forall S_{1}, S_{2} \in \operatorname{dom} f, \forall s \in S_{1} \triangle S_{2}, \exists t \in S_{1} \triangle S_{2}, f\left(S_{1} \triangle\{s, t\}\right)+f\left(S_{2} \triangle\{s, t\}\right) \geq f\left(S_{1}\right)+$ $f\left(S_{2}\right)$.

Note that $(V, \operatorname{dom} f)$ is a simultaneous delta-matroid.

For a weighted mixed graph $(G, w)$ with $G=(V, E, A)$ and $w \in \mathbf{R}^{E \cup A}$, define a function $f_{M F}$ : $2^{V} \rightarrow \mathbf{R} \cup\{-\infty\}$ by

$$
f_{M F}(S)= \begin{cases}\max \{w(F) \mid F \text { is a matching forest with } R(F)=S\} & \left(S \in \mathcal{F}_{M F}\right), \\ -\infty & \text { (otherwise). }\end{cases}
$$

We prove that $f_{M F}$ satisfies (S-VDM).

Theorem 2. For any weighted mixed graph $(G, w)$, it holds that $f_{M F}$ is a valuated delta-matroid.

Proofs for Theorems 1 and 2 will be given in $\S 2.2$. We remark that the relation between valuated delta-matroids in the sense of [6] and those in our sense is similar to that between M-concave functions and $\mathrm{M}^{\natural}$-concave functions [20].

The next contribution of this paper is new algorithms for the weighted matching forest problem: we design a simpler algorithm and a faster algorithm than Giles' algorithm [15]. In $\S 3$, we present a simple $\mathrm{O}\left(n^{2} m\right)$-time algorithm which focuses on the delta-matroid structure. We also present an $\mathrm{O}\left(n^{3}\right)$-time algorithm in $\S 4$ by using the technique of Gabow [13] for the weighted matching problem. 


\section{Delta-matroids and matching forests}

In this section, we prove Theorems 1 and 2. That is, we show relations between delta-matroids and matching forests, and between valuated delta-matroids and weighted matching forests.

\subsection{Matching delta-matroids and branching delta-matroids}

In this subsection, we describe basic facts on delta-matroids, including their relations to matchings and branchings. We begin with exhibiting two operations on delta-matroids. The dual of a deltamatroid $(V, \mathcal{F})$ is a delta-matroid $(V, \overline{\mathcal{F}})$, defined by $\overline{\mathcal{F}}=\{V \backslash S \mid S \in \mathcal{F}\}$. The union of two delta-matroids $\left(V, \mathcal{F}_{1}\right)$ and $\left(V, \mathcal{F}_{2}\right)$ is a pair $\left(V, \mathcal{F}_{1} \vee \mathcal{F}_{2}\right)$ defined by $\mathcal{F}_{1} \vee \mathcal{F}_{2}=\left\{S_{1} \cup S_{2} \mid S_{1} \in\right.$ $\left.\mathcal{F}_{1}, S_{2} \in \mathcal{F}_{2}, S_{1} \cap S_{2}=\emptyset\right\}$, which is a delta-matroid [2].

The relation between matchings and delta-matroids is well-known. Let $(G, w)$ be a weighted undirected graph with $G=(V, E)$ and $w \in \mathbf{R}^{E}$. As stated in $\S 1$, the pair $\left(V, \mathcal{F}_{M}\right)$, where $\mathcal{F}_{M}=\{\partial M \mid M$ is a matching in $G\}$, is an even delta-matroid, which we call the matching deltamatroid of $G$. Moreover, a function $f_{M}: 2^{V} \rightarrow \mathbf{R} \cup\{-\infty\}$ defined below is a valuated deltamatroid [19]:

$$
f_{M}(S)= \begin{cases}\max \{w(M) \mid M \text { is a matching with } \partial M=S\} & \left(S \in \mathcal{F}_{M}\right), \\ -\infty & \text { (otherwise) } .\end{cases}
$$

We now present a relation between branchings and delta-matroids. Let $(G, w)$ be a weighted directed graph with $G=(V, A)$ and $w \in \mathbf{R}^{A}$. Recall that $\mathcal{F}_{B}=\{R(B) \mid B$ is a branching in $G\}$. It is verified that $\left(V, \mathcal{F}_{B}\right)$ is a delta-matroid as follows. For a directed graph $G$, a strong component is called a source component if it has no arc entering from other strong components. The vertex set and arc set of a strong component $K$ are denoted by $V K$ and $A K$, respectively. Let $K_{1}, \ldots, K_{l}$ be all source components in $G$. Then, we have that $\mathcal{F}_{B}=\left\{S|S \subseteq V|, S \cap V K_{i} \mid \geq 1\right.$ for $\left.i=1, \ldots, l\right\}$. Thus, it follows that $\left(V, \mathcal{F}_{B}\right)$ is a generalized matroid [12]. Moreover, it also follows that $\left(V, \mathcal{F}_{B}\right)$ satisfies $(\mathrm{SDM})$. We call $\left(V, \mathcal{F}_{B}\right)$ as the branching delta-matroid of $G$.

Theorem 3. For any directed graph $G$, it holds that $\left(V, \mathcal{F}_{B}\right)$ is a simultaneous delta-matroid.

Furthermore, this fact extends to weighted branchings. Define $f_{B}: 2^{V} \rightarrow \mathbf{R} \cup\{-\infty\}$ by

$$
f_{B}(S)= \begin{cases}\max \{w(B) \mid B \text { is a branching with } R(B)=S\} & \left(S \in \mathcal{F}_{B}\right), \\ -\infty & \text { (otherwise) }\end{cases}
$$

Then, $f_{B}$ is a valuated delta-matroid, which immediately follows from arguments in Schrijver [21, Theorem 1].

Theorem 4. For any weighted directed graph $(G, w)$, it holds that $f_{B}$ is a valuated delta-matroid.

\subsection{Delta-matroids and matching forests}

In this subsection, we prove Theorems 1 and 2. We begin with a simple proof showing that $\left(V, \mathcal{F}_{M F}\right)$ is a delta-matroid for a mixed graph $(V, E, A)$. Let $\mathcal{F}_{M}$ be the matching delta-matroid of $(V, E)$ and $\mathcal{F}_{B}$ the branching delta-matroid of $(V, A)$. Then, it immediately follows from the definition of matching forests that $\mathcal{F}_{M F}$ is the dual of $\mathcal{F}_{M} \vee \overline{\mathcal{F}}_{B}$, and thus $\left(V, \mathcal{F}_{M F}\right)$ is a delta-matroid.

We now prove Theorem 1, which is a stronger statement. First, Schrijver [21] proved the following exchange property of branchings. 
Lemma 5 (Schrijver [21]). Let $G=(V, A)$ be a directed graph, and $B_{1}$ and $B_{2}$ be branchings partitioning $A$. Let $R_{1}$ and $R_{2}$ be vertex sets with $R_{1} \cup R_{2}=R\left(B_{1}\right) \cup R\left(B_{2}\right)$ and $R_{1} \cap R_{2}=$ $R\left(B_{1}\right) \cap R\left(B_{2}\right)$. Then $A$ can be split into branchings $B_{1}^{\prime}$ and $B_{2}^{\prime}$ with $R\left(B_{i}^{\prime}\right)=R_{i}$ for $i=1,2$ if and only if each source component $K$ in $G$ satisfies that $\left|K \cap R_{i}\right| \geq 1$ for $i=1,2$.

By using Lemma 5, Schrijver proved an exchange property of matching forests [21, Theorem 2]. Here, we show another exchange property of matching forests, which relates them to simultaneous delta-matroids. The proof below is quite similar to the proof for Theorem 2 in [21]. For completeness, however, we describe a full proof.

Lemma 6. Let $G=(V, E, A)$ be a mixed graph, $F_{1}$ and $F_{2}$ be matching forests partitioning $E \cup A$, and $s \in R\left(F_{2}\right) \backslash R\left(F_{1}\right)$. Then, there exist matching forests $F_{1}^{\prime}$ and $F_{2}^{\prime}$ which partition $E \cup A$ and satisfy one of the following:

(i) $R\left(F_{1}^{\prime}\right)=R\left(F_{1}\right) \cup\{s\}$ and $R\left(F_{2}^{\prime}\right)=R\left(F_{2}\right) \backslash\{s\}$,

(ii) $R\left(F_{1}^{\prime}\right)=R\left(F_{1}\right) \cup\{s, t\}$ and $R\left(F_{2}^{\prime}\right)=R\left(F_{2}\right) \backslash\{s, t\}$ for some $t \in R\left(F_{2}\right) \backslash\left(R\left(F_{1}\right) \cup\{s\}\right)$,

(iii) $R\left(F_{1}^{\prime}\right)=\left(R\left(F_{1}\right) \cup\{s\}\right) \backslash\{t\}$ and $R\left(F_{2}^{\prime}\right)=\left(R\left(F_{2}\right) \backslash\{s\}\right) \cup\{t\}$ for some $t \in R\left(F_{1}\right) \backslash R\left(F_{2}\right)$.

Proof. Let $M_{i}:=F_{i} \cap E$ and $B_{i}:=F_{i} \cap A$ for $i=1,2$. Denote the family of the source components in $(V, A)$ by $\mathcal{K}$. If $v \in R\left(B_{1}\right) \cap R\left(B_{2}\right)$ for $v \in V$, then we have $\{v\} \in \mathcal{K}$. Thus, for a source component $K \in \mathcal{K}$ with $|K| \geq 2, K \cap R\left(B_{1}\right)$ and $K \cap R\left(B_{2}\right)$ are not empty and disjoint with each other. For each $K \in \mathcal{K}$ with $|K| \geq 2$, choose a pair $e_{K}$ of vertices, one of which is in $K \cap R\left(B_{1}\right)$ and the other in $K \cap R\left(B_{2}\right)$. Denote $N=\left\{e_{K} \mid K \in \mathcal{K}\right\}$. Note that $N$ is a matching.

Construct an undirected graph $H=\left(V, M_{1} \cup M_{2} \cup N\right)$. We have that $H$ is a disjoint collection of paths and cycles. For, an endpoint $u$ of an edge $e_{K} \in N$ satisfies that either $u \in \partial^{-} B_{1}$ or $u \in \partial^{-} B_{2}$, and thus $u$ is not covered by both of $M_{1}$ and $M_{2}$. Moreover, we have that $s$ is an endpoint of a path $P$ in $H$. For, since $s \in R\left(F_{2}\right)$, we have that $s$ is not covered by $M_{2}$. If $s$ is covered by $M_{1}$, then $s \in R\left(B_{1}\right)$, and thus $s \in R\left(B_{1}\right) \cap R\left(B_{2}\right)$. This implies that $s$ is not covered by $N$.

Denote the set of vertices on $P$ by $V P$, the set of edges in $M_{1} \cup M_{2}$ on $P$ by $E P$, and let $M_{1}^{\prime}:=M_{1} \triangle E P$ and $M_{2}^{\prime}:=M_{2} \triangle E P$. Then, both $M_{1}^{\prime}$ and $M_{2}^{\prime}$ are matchings and

$$
R\left(M_{1}^{\prime}\right)=\left(R\left(M_{1}\right) \backslash V P\right) \cup\left(R\left(M_{2}\right) \cap V P\right), \quad R\left(M_{2}^{\prime}\right)=\left(R\left(M_{2}\right) \backslash V P\right) \cup\left(R\left(M_{1}\right) \cap V P\right) .
$$

Now, by Lemma 5 , there exist disjoint branchings $B_{1}^{\prime}$ and $B_{2}^{\prime}$ such that

$$
R\left(B_{1}^{\prime}\right)=\left(R\left(B_{1}\right) \backslash V P\right) \cup\left(R\left(B_{2}\right) \cap V P\right), \quad R\left(B_{2}^{\prime}\right)=\left(R\left(B_{2}\right) \backslash V P\right) \cup\left(R\left(B_{1}\right) \cap V P\right) .
$$

(Note that $\left|K \cap R\left(B_{i}^{\prime}\right)\right| \geq 1$ for $i=1,2$ for every source component $K$.)

Since $R\left(B_{i}\right) \cup R\left(M_{i}\right)=V$, we have that $F_{i}^{\prime}:=B_{i}^{\prime} \cup M_{i}^{\prime}$ is a matching forest for $i=1,2$, and

$$
R\left(F_{1}^{\prime}\right)=\left(R\left(F_{1}\right) \backslash V P\right) \cup\left(R\left(F_{2}\right) \cap V P\right), \quad R\left(F_{2}^{\prime}\right)=\left(R\left(F_{2}\right) \backslash V P\right) \cup\left(R\left(F_{1}\right) \cap V P\right) .
$$

If $V P=\{s\}$, then Assertion (i) applies. Otherwise, denote the other endpoint of $P$ by $t$. If $t \in V \backslash\left(R\left(F_{1}\right) \triangle R\left(F_{2}\right)\right)$, then Assertion (i) applies. If $t \in R\left(F_{2}\right) \backslash R\left(F_{1}\right)$, then Assertion (ii) applies. If $t \in R\left(F_{1}\right) \backslash R\left(F_{2}\right)$, then Assertion (iii) applies.

Theorem 1 is obvious from Lemma 6 . Furthermore, Theorem 2 also follows from Lemma 6. Proof for Theorem 2. Let $S_{1}, S_{2} \in \operatorname{dom} f$ and $s \in S_{1} \triangle S_{2}$. For $i=1,2$, let $F_{i}$ be a matching forest such that $R\left(F_{i}\right)=S_{i}$ and $w\left(F_{i}\right)=f_{M F}\left(S_{i}\right)$. Without loss of generality, assume $s \in R\left(F_{2}\right) \backslash R\left(F_{1}\right)$. By applying Lemma 6 to the mixed graph consisting of the edges in $F_{1}$ and $F_{2}$, we obtain matching forests $F_{1}^{\prime}$ and $F_{2}^{\prime}$ such that $w\left(F_{1}^{\prime}\right)+w\left(F_{2}^{\prime}\right)=w\left(F_{1}\right)+w\left(F_{2}\right)$ and satisfying one of Assertions (i)-(iii). Now the statement follows from $w\left(F_{1}^{\prime}\right) \leq f_{M F}\left(R\left(F_{1}^{\prime}\right)\right)$ and $w\left(F_{2}^{\prime}\right) \leq f_{M F}\left(R\left(F_{2}^{\prime}\right)\right)$. 


\section{A simpler algorithm}

Let $(G, w)$ be a weighted mixed graph with $G=(V, E, A)$ and $w \in \mathbf{R}^{E \cup A}$. In this section, we describe a primal-dual algorithm for finding a matching forest $F$ maximizing $w(F)$. This algorithm is a slight modification of Giles' algorithm [15]. The main difference results from focusing the delta-matroid structure of branchings (Theorem 3).

\subsection{LP formulation for the weighted matching forest problem}

For a subpartition $\mathcal{L}$ of $V$, let $\cup \mathcal{L}$ denote the union of the sets in $\mathcal{L}$ and let

$$
\gamma(\mathcal{L}):=\{e \mid e \in E, e \text { is contained in } \cup \mathcal{L}\} \cup\{a \mid a \in A, a \text { is contained in some set in } \mathcal{L}\} .
$$

Let $\Lambda$ denote the collection of subpartition $\mathcal{L}$ of $V$ with $|\mathcal{L}|$ odd. The following is a linear programming relaxation of an integer program describing the weighted matching forest problem:

$$
\begin{array}{lll}
\text { (P) maximize } & \sum_{e \in E \cup A} w(e) x(e) & \\
\text { subject to } & x\left(\delta^{\text {head }}(v)\right) \leq 1 & (v \in V), \\
& x(\gamma(\mathcal{L})) \leq\lfloor|\cup \mathcal{L}|-|\mathcal{L}| / 2\rfloor & (\mathcal{L} \in \Lambda), \\
& x(e) \geq 0 & (e \in E \cup A) .
\end{array}
$$

Here, $\delta^{\text {head }}(v) \subseteq E \cup A$ denotes the set of edges which have $v$ as a head, i.e., $\delta^{\text {head }}(v)=\delta v \cup$ $\delta^{-} v$. Note that the above linear system is a common extension of those describing the weighted matching problem [7] and the weighted branching problem [9]. Giles [15] proved the integrality of the system (1)-(3).

Theorem 7 ([15]). For any weighted mixed graph $(G, w)$, the linear program $(\mathrm{P})$ has an integer optimal solution.

Furthermore, Schrijver [21] proved that the system (1)-(3) is totally dual integral [10], which commonly extends the total dual integrality of those for matchings [4] and for branchings. That is, Schrijver proved that the following dual problem of $(\mathrm{P})$ has an integer optimal solution if $w$ is integer:

$$
\begin{aligned}
& \text { (D) } \quad \text { minimize } \quad \sum_{v \in V} y(v)+\sum_{\mathcal{L} \in \Lambda} z(\mathcal{L})\lfloor|\cup \mathcal{L}|-|\mathcal{L}| / 2\rfloor \\
& \text { subject to } y(u)+y(v)+\sum_{\mathcal{L}: e \in \gamma(\mathcal{L})} z(\mathcal{L}) \geq w(e) \quad(e=u v \in E) \text {, } \\
& y(v)+\sum_{\mathcal{L}: a \in \gamma(\mathcal{L})} z(\mathcal{L}) \geq w(a) \quad(a=u v \in A), \\
& y(v) \geq 0 \quad(v \in V), \\
& z(\mathcal{L}) \geq 0 \quad(\mathcal{L} \in \Lambda) .
\end{aligned}
$$

Theorem 8 ([21]). For any weighted mixed graph $(G, w)$ with $w$ integer, the linear program (D) has an integer optimal solution. 
Define the reduced weight $w^{\prime} \in \mathbf{R}^{E \cup A}$ by

$$
\begin{array}{ll}
w^{\prime}(e)=y(u)+y(v)+\sum_{\mathcal{L}: e \in \gamma(\mathcal{L})} z(\mathcal{L})-w(e) & (e=u v \in E), \\
w^{\prime}(a)=y(v)+\sum_{\mathcal{L}: a \in \gamma(\mathcal{L})} z(\mathcal{L})-w(a) & (a=u v \in A) .
\end{array}
$$

Below are the complementary slackness conditions of $(\mathrm{P})$ and $(\mathrm{D})$.

$$
\begin{array}{rlrl}
x(e)>0 & \Longrightarrow w^{\prime}(e)=0 & & (e \in E \cup A), \\
x\left(\delta^{\text {head }} v\right)<1 & \Longrightarrow y(v)=0 & (v \in V), \\
z(\mathcal{L})>0 & \Longrightarrow x(\gamma(\mathcal{L}))=\lfloor|\cup \mathcal{L}|-|\mathcal{L}| / 2\rfloor & (\mathcal{L} \in \Lambda) .
\end{array}
$$

\subsection{Algorithm description}

\subsubsection{Notations}

In the algorithm, we keep a matching forest $F$, which corresponds to an integer feasible solution $x$ of $(\mathrm{P})$, and a dual feasible solution $(y, z)$. We maintain that $x$ and $(y, z)$ satisfy $(8)$ and $(10)$. The algorithm terminates when (9) is satisfied.

Similarly to the classical weighted matching and branching algorithms, we execute shrinking of subgraphs repeatedly. We keep two laminar families $\Delta$ and $\Upsilon$ of subsets of $V$, the former of which results from shrinking a strong component in the directed graph and the latter from shrinking an undirected odd cycle.

We use the following notations to describe the algorithm.

- For a cycle or a path $Q$ in an undirected graph $(V, E)$, let $V Q$ and $E Q$ denote the vertex set and edge set of $Q$, respectively. We often abbreviate $E Q$ as $Q$.

- $\Omega^{\prime}:=\Delta \cup \Upsilon, \Omega:=\Omega^{\prime} \cup\{\{v\} \mid v \in V\}$.

- For each $U \in \Omega^{\prime}$, let $G_{U}=\left(V_{U}, E_{U}, A_{U}\right)$ denote the mixed graph obtained from the subgraph induced by $U$ by contracting all maximal proper subsets of $U$ belonging to $\Delta$. Also, let $\hat{G}=(\hat{V}, \hat{E}, \hat{A})$ denote the mixed graph obtained from $G$ by contracting all maximal sets in $\Omega^{\prime}$. We denote a vertex in a shrunk graph by the set of vertices in $V$ which are shrunk into the vertex. Also, we often identify a vertex $U$ in a shrunk graph and the singleton $\{U\}$.

- For $G=(V, E, A)$ and a dual feasible solution $(y, z)$, the equality subgraph $G^{\circ}=\left(V, E^{\circ}, A^{\circ}\right)$ of $G$ is a subgraph defined by $E^{\circ}=\left\{e \mid e \in E, w^{\prime}(e)=0\right\}$ and $A^{\circ}=\left\{a \mid a \in A, w^{\prime}(a)=0\right\}$. We denote the branching delta-matroid in $\left(\hat{V}, \hat{A}^{\circ}\right)$ by $\left(\hat{V}, \hat{\mathcal{F}}_{B}^{\circ}\right)$, i.e.,

$$
\hat{\mathcal{F}}_{B}^{\circ}=\left\{R(\hat{B}) \mid \hat{B} \subseteq \hat{A}^{\circ} \text { is a branching in } \hat{G}^{\circ}\right\} .
$$

The outline of the algorithm is as follows.

- We maintain a matching forest $\hat{F}=\hat{M} \cup \hat{B}$ in $\hat{G}^{\circ}$, where $\hat{M} \subseteq \hat{E}^{\circ}$ and $\hat{B} \subseteq \hat{A}^{\circ}$, in order to maintain (8).

- In contracting a vertex set $U \subseteq V$, we associate a partition $\mathcal{L}_{U}$ of $U$ such that $x\left(\gamma\left(\mathcal{L}_{U}\right)\right)=$ $\left\lfloor\left|\cup \mathcal{L}_{U}\right|-\left|\mathcal{L}_{U}\right| / 2\right\rfloor$. The vector $z$ is restricted to subpartitions associated to the sets in $\Omega^{\prime}$ in order to maintain (10). 

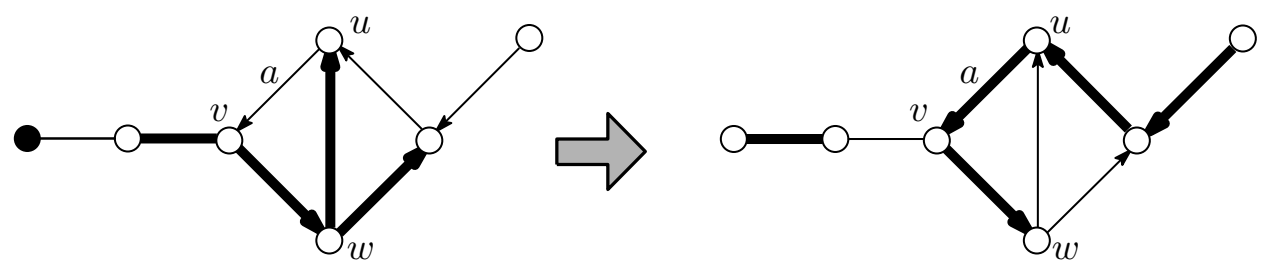

Figure 1: Augmentation. (Thick edges are in $\hat{F}$ and the black vertex is a source vertex.)

- Similarly to Edmonds' matching algorithm [8], we construct an alternating forest $H$, which is a subgraph of $\left(\hat{V}, \hat{E}^{\circ}\right)$. The vertex set and edge set of $H$ are denoted by $\hat{V} H$ and $\hat{E} H$, respectively. We often abbreviate $\hat{E} H$ as $H$. Each component of $H$ is a tree and contains a unique source vertex. Intuitively, a source vertex is a vertex where (9) is not satisfied (see $\S 3.2 .3$ for precise definition). For $v \in \hat{V} H$, let $P_{v}$ denote the path in $H$ connecting a source vertex and $v$. The edges incident to a source vertex does not belong to $\hat{M}$, and edges in $\hat{M}$ and $\hat{E}^{\circ} \backslash \hat{M}$ appear alternately on each $P_{v}$. We label a vertex $v$ as "even" (resp., "odd") if the length of $P_{v}$ is even (resp., odd). Here, the length of a path is defined by the number of its edges. The set of vertices labelled as even (resp., odd) is denoted by even $(H)$ (resp., $\operatorname{odd}(H))$. Also, let free $(H):=\hat{V} \backslash(\operatorname{even}(H) \cup \operatorname{odd}(H))$.

\subsubsection{A rough description of augmentation and shrinking}

Before presenting a full description of the algorithm, we briefly sketch how to augment the matching forest, shrink subgraphs and associate a partition with the shrunk vertex set.

To make things easy, let us suppose that no subgraph is shrunk, i.e., $\Delta=\Upsilon=\emptyset$ and $\hat{G}=G$. Denote the current matching forest by $\hat{F}=\hat{M} \cup \hat{B}$, where $\hat{M} \subseteq \hat{E}^{\circ}$ is a matching and $\hat{B} \subseteq \hat{A}^{\circ}$ is a branching.

After labeling a vertex $v$ as even in growing the alternating forest $H$, we search for an arc $a \in$ $\hat{A}^{\circ} \cap \delta^{-} v$. Note that arcs in $\delta^{-} v$ do not belong to $\hat{B}$. If such an arc $a$ is found, our algorithm proceeds as follows.

- If $R(\hat{B}) \backslash\{v\} \in \hat{\mathcal{F}}_{B}^{\circ}$, then reset the matching forest $\hat{F}:=M^{\prime} \cup B^{\prime}$, where $M^{\prime}:=\hat{M} \triangle P_{v}$ and $B^{\prime}$ is a branching in $\left(\hat{V}, \hat{A}^{\circ}\right)$ with $R\left(B^{\prime}\right)=R(\hat{B}) \backslash\{v\}$. This procedure is one kind of augmentation, in which the number of vertices violating (9) decreases. See Figure 1 for an illustration.

- If $R(\hat{B}) \backslash\{v\} \notin \hat{\mathcal{F}}_{B}^{\circ}$, it follows that $v \in \hat{V} K$ for some source component $K$ of $\left(\hat{V}, \hat{A}^{\circ}\right)$ and $\hat{V} K \backslash\{v\} \subseteq \partial^{-} \hat{B}$. If $K$ contains no undirected edge in $\hat{E}^{\circ}$, we add $X=\hat{V} K$ to $\Delta$ and update $\hat{G}$ by contracting the vertices in $X$ to a single vertex. The newly created vertex is called a pseudo-vertex, denoted by $X \in \hat{V}$. We then define a partition $\mathcal{L}_{X}$ of $X$ by $\mathcal{L}_{X}=\{X\}$ and set $z\left(\mathcal{L}_{X}\right)=0$. Note that $(10)$ holds for $\mathcal{L}_{X}$, since $x\left(\gamma\left(\mathcal{L}_{X}\right)\right)=|X|-1,\left|\cup \mathcal{L}_{X}\right|=|X|$ and $\left|\mathcal{L}_{X}\right|=1$. See Figure 2 for an illustration. The case where $K$ contains an undirected edge in $\hat{E}^{\circ}$ will be described in $\S 3.2 .3$.

Note that we can determine whether $R(\hat{B}) \backslash\{v\} \in \hat{\mathcal{F}}_{B}^{\circ}$ or not by decomposing $\left(\hat{V}, \hat{A}^{\circ}\right)$ into strong components. Also, it is not difficult to find a branching $B^{\prime} \subseteq \hat{A}^{\circ}$ with $R\left(B^{\prime}\right)=R(B) \backslash\{v\}$.

Remark 9. The above bifurcation is the main difference from Giles' algorithm [15]. In Giles' algorithm, we augment the matching forest if $B^{\prime \prime}=\hat{B} \cup\{a\}$ is a branching in $\hat{G}$, which is a 


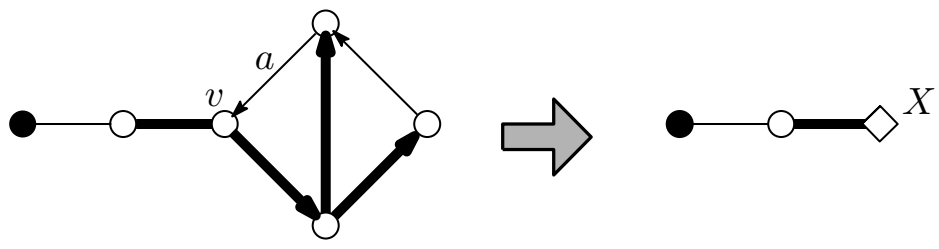

Figure 2: Shrinking of a source component. (Thick edges are in $\hat{F}$ and the black vertex is a source vertex. The square in the shrunk graph indicates the pseudo-vertex $X \in \hat{V}$.)
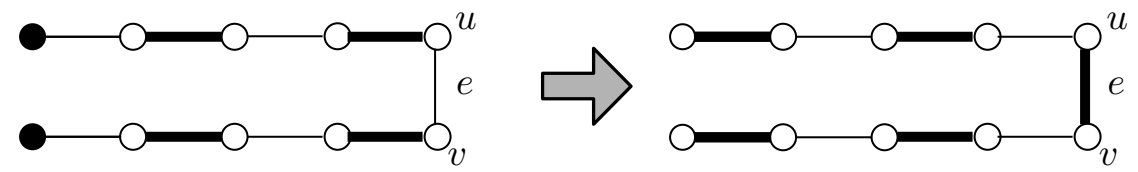

Figure 3: Augmentation. (Thick edges are in $\hat{F}$ and the black vertex is a source vertex.)

sufficient condition for $R(\hat{B}) \backslash\{v\} \in \hat{\mathcal{F}}_{B}^{\circ}$. (Here, we reset the matching forest $\hat{F}:=M^{\prime} \cup B^{\prime \prime}$.) If $B^{\prime \prime}$ is not a branching, we have that $B^{\prime \prime}$ contains exactly one directed cycle $D$, and we contract $X^{\prime}=V D$ to a single vertex. For instance, in Figure 1 we do not augment $\hat{F}$ but contract the directed cycle consisting of $u, v, w$ in Giles' algorithm.

Also, if we find an undirected edge $e \in \hat{E}^{\circ}$ connecting two even vertices $u$ and $v$, we do similar procedures as the the classical blossom algorithm [8]. If $u$ and $v$ belong to different components in $H$, then we augment the matching forest by resetting $\hat{F}:=M^{\prime} \cup \hat{B}$, where $M^{\prime}:=M \triangle\left(P_{u} \cup P_{v} \cup\{e\}\right)$. See Figure 3 for an illustration.

Assume that $u$ and $v$ belong to the same component of $H$ (see Figure 4 for an illustration). Here, $H \cup\{e\}$ contains exactly one odd undirected cycle $C$. We now add $U=\hat{V} C$ to $\Upsilon$, and update $\hat{G}$ by contracting the vertices in $U$ to a pseudo-vertex $U \in \hat{V}$. We then define a partition $\mathcal{L}_{U}$ of $U$ by $\mathcal{L}_{U}=\{\{v\} \mid v \in U\}$ and set $z\left(\mathcal{L}_{U}\right)=0$. Note that $(10)$ holds for $\mathcal{L}_{U}$, since $x\left(\gamma\left(\mathcal{L}_{U}\right)\right)=\lfloor|U| / 2\rfloor$ and $\left|\cup \mathcal{L}_{U}\right|=\left|\mathcal{L}_{U}\right|=|U|$.

Dealing with a subgraph containing pseudo-vertices is a bit more complicated. Consider shrinking a source component $K$ in $\hat{G}$ and let $X \subseteq V$ be the union of vertices in $\hat{V} K$. Denote the maximal

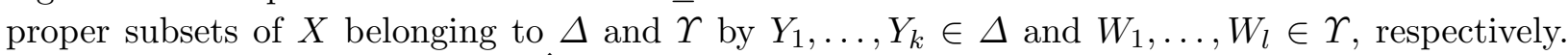
Here, we can assume that arcs in $\hat{A} K \cap\left(\delta^{+} W_{i} \cup \delta^{-} W_{i}\right)$ are incident to an identical vertex $v_{W_{i}} \in V_{W_{i}}$ for every $i=1, \ldots, l$, since otherwise we can augment the current matching forest (see Figure 5). Let $X^{\prime}=\left(X \backslash\left(W_{1} \cup \cdots \cup W_{l}\right)\right) \cup\left\{v_{W_{1}}\right\} \cup \cdots \cup\left\{v_{W_{l}}\right\}$. Note that $X^{\prime}$ forms the vertex set of a strong component in $\left(V, A^{\circ}\right)$. We now add $X^{\prime}$ to $\Delta$ and let $\mathcal{L}_{X^{\prime}}=\left\{X^{\prime}\right\}$ be the associated partition with

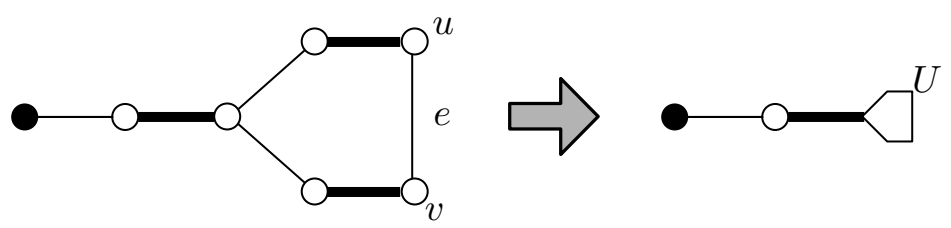

Figure 4: Shrinking of an undirected odd cycle. (Thick edges are in $\hat{F}$ and the black vertex is a source vertex. The pentagon in the shrunk graph indicates the pseudo-vertex $U \in \hat{V}$.) 

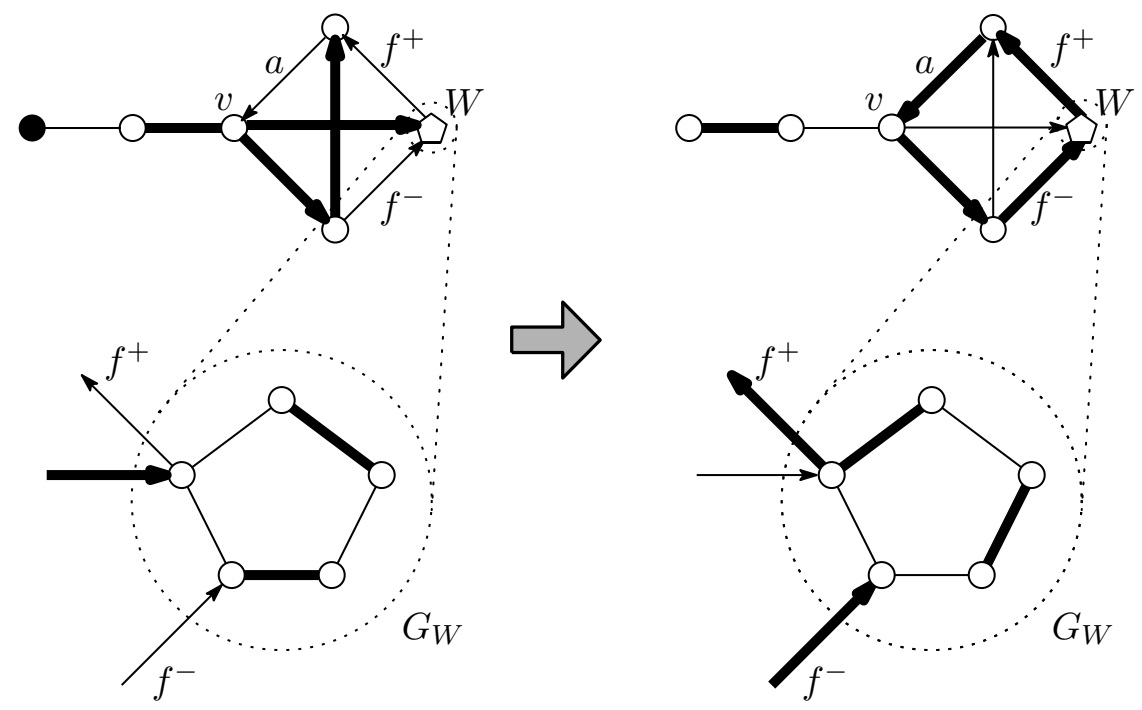

Figure 5: Augmentation. (Thick edges are in $\hat{F}$ and the black vertex is a source vertex. The graph inside the dotted circle indicates $G_{W}$, which is shrunk into the vertex $W$ in $\hat{G}^{\circ}$.)

$X^{\prime}$. See Figure 6 for an illustration.

Finally, consider shrinking an odd undirected cycle $C$. Let $U \subseteq V$ denote the union of vertices in $\hat{V} C$. Denote the maximal proper subsets of $U$ belonging to $\Delta$ by $Y_{1} \ldots, Y_{k} \in \Delta$ and the proper subsets of $U$ belonging to $\Upsilon W_{1} \ldots, W_{l} \in \Upsilon$, respectively. Let $C_{U}$ be an odd cycle in $G_{U}$ which can be obtained by adding even number of edges from each $E_{W_{j}}$ to $C$. For $i=1, \ldots, k$, let $f_{i}^{1}, f_{i}^{2} \in C_{U}$ denote the two edges incident to $Y_{i}$, and let $v_{i}^{1}, v_{i}^{2} \in Y_{i}$ denote the vertices to which $f_{i}^{1}$ and $f_{i}^{2}$ are incident, respectively. If, for some $Y_{i}$, the two vertices $v_{i}^{1}$ and $v_{i}^{2}$ are distinct and $z\left(\mathcal{L}_{Y_{i}^{\prime}}\right)=0$ for the minimal subset $Y_{i}^{\prime} \in \Delta$ of $Y_{i}$ such that $\left\{v_{i}^{1}, v_{i}^{2}\right\} \subseteq Y_{i}^{\prime}$, we can augment the current matching forest (see Figure 7 for an illustration).

Suppose otherwise. Without loss of generality, assume that $v_{i}^{1}$ and $v_{i}^{2}$ are identical for $i=$ $1, \ldots, j$, and distinct for $i=j+1, \ldots, k$. For $i=j+1, \ldots, k$, let $Y_{i}^{\prime} \in \Delta$ be the minimal subset of $Y_{i}$ such that $\left\{v_{i}^{1}, v_{i}^{2}\right\} \subseteq Y_{i}^{\prime}$. Note that $z\left(\mathcal{L}_{Y_{i}^{\prime}}\right)>0$ for $i=j+1, \ldots, k$. Let $U^{\prime}=$ $\left(U \backslash\left(Y_{1} \cup \cdots \cup Y_{k}\right)\right) \cup\left\{v_{Y_{1}}, \ldots, v_{Y_{j}}\right\} \cup\left(Y_{j+1}^{\prime} \cup \cdots Y_{k}^{\prime}\right)$. We now add $U^{\prime}$ to $\Upsilon$, and define a partition $\mathcal{L}_{U^{\prime}}$ of $U^{\prime}$ by the collection of $\left\{v_{Y_{1}}\right\}, \ldots,\left\{v_{Y_{j}}\right\}, Y_{j+1}^{\prime}, \ldots, Y_{k}^{\prime}$ and singletons of the other vertices in $U^{\prime}$. See Figure 8 for an illustration.

\subsubsection{A full description of the algorithm}

We now present a full description of our algorithm.

\section{Algorithm SIMPLE}

Input. A weighted mixed graph $(G, w)$, where $G=(V, E, A)$ and $w \in \mathbf{R}^{E \cup A}$.

Output. A matching forest $F$ in $G$ maximizing $w(F)$.

Step 1. Set $F:=\emptyset, y(v):=\max \{\{w(e) / 2 \mid e \in E\},\{w(a) \mid a \in A\}\}$ for every $v \in V, \Delta:=\emptyset$ and $\Upsilon:=\emptyset$. (Hence $\Omega=\{\{v\} \mid v \in V\}, \hat{G}=G, \hat{F}=\emptyset$ and $z$ is void.)

Step 2. Construct the equality subgraph $\hat{G}^{\circ}=\left(\hat{V}, \hat{E}^{\circ}, \hat{A}^{\circ}\right)$. Define the set of source vertices $\hat{S}=$ $\left\{U \mid U \in \hat{V}, y(v)>0\right.$ and $x\left(\delta^{\text {head }}(v)\right)=0$ for some $\left.v \in U\right\}$. If $\hat{S}=\emptyset$, deshrink every sets in 

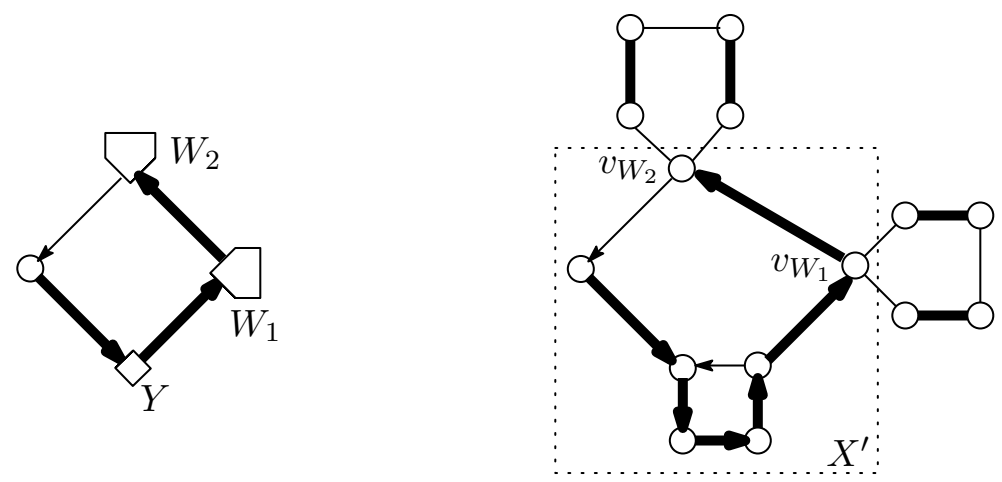

Figure 6: The graph on the left is a strong component $K$ in $\hat{G}$, where $Y \in \Delta$ and $W_{1}, W_{2} \in \Upsilon$. The graph on the right represents a graph obtained by deshrinking $Y, W_{1}$ and $W_{2}$. The dotted squares indicate $X^{\prime}$, which is newly added to $\Delta$.
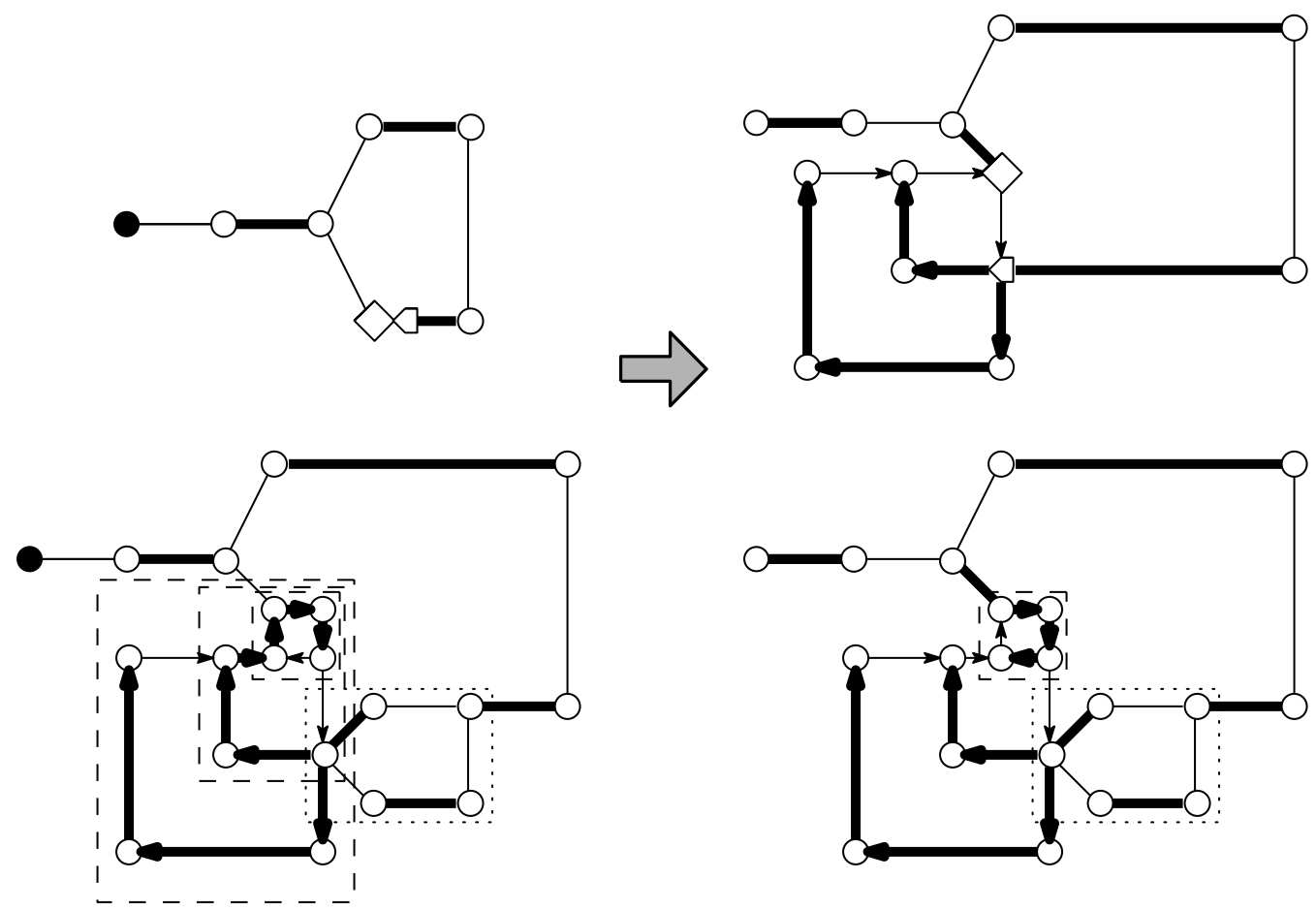

Figure 7: The two graphs above are $\hat{G}$ and those below are $G$. The dotted box indicates $W \in \Upsilon$ and the nested three dashed boxes indicate $Y, Y^{\prime}, Y^{\prime \prime} \in \Delta$, where $Y^{\prime \prime} \subseteq Y^{\prime} \subseteq Y$ and $z\left(\mathcal{L}_{Y^{\prime}}\right)=z\left(\mathcal{L}_{Y}\right)=0$. In the present step, augmentation and deshrinking of $Y$ and $Y^{\prime}$ are executed. 

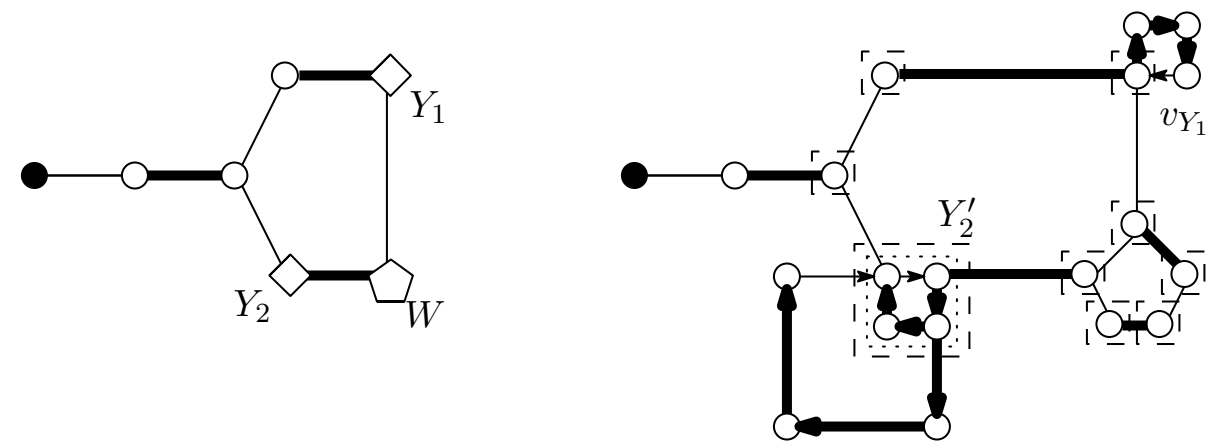

Figure 8: The graph on the left is an odd cycle $C$ in $\hat{G}$, where $Y_{1}, Y_{2} \in \Delta$ and $W \in \Upsilon$. The graph in the right represents $G$, where the dotted box indicates $Y_{2}^{\prime}$ with $z\left(\mathcal{L}_{Y_{2}^{\prime}}\right)>0$. The set of vertices inside the dashed boxes is $U^{\prime}$, where the dashed boxes indicate the partition $\mathcal{L}_{U^{\prime}}$.
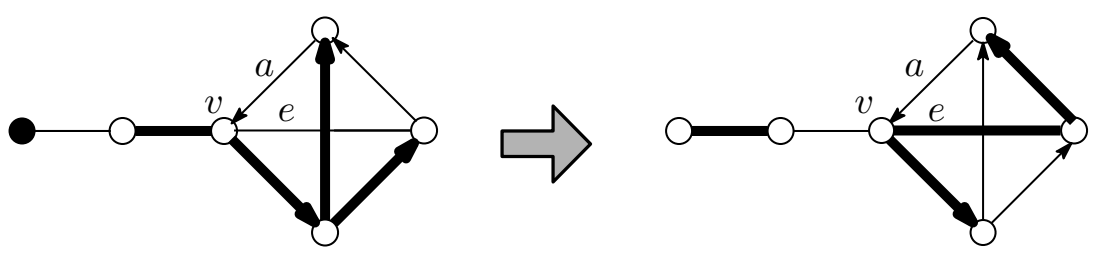

Figure 9: Augmentation in Step 4.2.1. (Thick edges are in $\hat{F}$ and the black vertex is a source vertex.)

$\Omega^{\prime}$ and return $F$. Otherwise, let $H$ be $(\hat{S}, \emptyset)$, label the vertices in $\hat{S}$ as even, and then go to Step 3.

Step 3. If there exists an arc $a \in \hat{A}^{\circ} \backslash \hat{B}$ with $\partial^{-} a \in \operatorname{even}(H)$, then go to Step 4. Otherwise, go to Step 5 .

Step 4. Let $v:=\partial^{-} a$. If $R(\hat{B}) \backslash\{v\} \in \hat{\mathcal{F}}_{B}^{\circ}$, then go to Step 4.1. Otherwise, go to Step 4.2.

Step 4.1: Augmentation. Reset $\hat{F}:=M^{\prime} \cup B^{\prime}$, where $M^{\prime}:=\hat{M} \triangle P_{v}$ and $B^{\prime}$ is a branching in $\left(\hat{V}, \hat{A}^{\circ}\right)$ with $R\left(B^{\prime}\right)=R(\hat{B}) \backslash\{v\}$. Delete each $T \in \Omega^{\prime}$ with $z\left(\mathcal{L}_{T}\right)=0$ from $\Omega^{\prime}$, and then go to Step 2. See Figure 1 for an illustration.

Step 4.2. Let $K$ be the source component containing $v$ and let $X \subseteq V$ be the union of vertices in $\hat{V} K$.

- If there exists $e \in \hat{E}^{\circ} \backslash \hat{M}$ such that $\partial e \subseteq \hat{V} K$, then go to Step 4.2.1.

- Otherwise, go to Step 4.2.2.

Step 4.2.1: Augmentation. Let $B_{K}$ be a branching in $K$ with $R\left(B_{K}\right)=\partial e$. Reset $\hat{F}:=M^{\prime} \cup B^{\prime}$, where $M^{\prime}:=\left(\hat{M} \triangle P_{v}\right) \cup\{e\}$ and $B^{\prime}:=(\hat{B} \backslash \hat{A} K) \cup B_{K}$, delete each $T \in \Omega^{\prime}$ with $z\left(\mathcal{L}_{T}\right)=0$ from $\Omega^{\prime}$, and then go to Step 2. See Figure 9 for an illustration.

Step 4.2.2. Let $W_{1}, \ldots, W_{l}$ be the maximal proper subsets of $X$ belonging to $\Upsilon$. If, for some $i \in\{1, \ldots, l\}, \hat{A} K$ contains a pair of $\operatorname{arcs} f^{+} \in \delta^{+} W_{i}$ and $f^{-} \in \delta^{-} W_{i}$ such that $\partial^{+} f^{+}$and $\partial^{-} f^{-}$belong to distinct vertices in $G_{W_{i}}$, then go to Step 4.2.2.1. Otherwise, go to Step 4.2.2.2. 
Step 4.2.2.1: Augmentation. Let $B_{K}$ be a branching in $K$ such that $R\left(B_{K}\right)=\left\{W_{i}\right\}$ and $f^{+} \in$ $B_{K}$. Reset $\hat{F}:=M^{\prime} \cup B^{\prime}$, where $M^{\prime}:=\hat{M} \triangle P_{v}$ and $B^{\prime}:=(\hat{B} \backslash \hat{A}(K)) \cup B_{K} \cup\left\{f^{-}\right\}$. Then, delete each $T \in \Omega^{\prime}$ with $z\left(\mathcal{L}_{T}\right)=0$ from $\Omega^{\prime}$ and go to Step 2. See Figure 5 for an illustration.

Step 4.2.2.2: Shrinking. For each $i=1, \ldots, l$, let $v_{W_{i}} \in V_{W_{i}}$ denote the unique vertex in $G_{W_{i}}$ to which arcs in $\hat{A} K$ are incident. Let $X^{\prime}=\left(X \backslash\left(W_{1} \cup \cdots \cup W_{l}\right)\right) \cup\left\{v_{W_{i}}\right\} \cup \cdots \cup\left\{v_{W_{l}}\right\}$ and add $X^{\prime}$ to $\Delta$. Let $\mathcal{L}_{X^{\prime}}=\left\{X^{\prime}\right\}$ be the associated partition with $X^{\prime}$, set $z\left(\mathcal{L}_{X^{\prime}}\right):=0$, and then go to Step 3. See Figure 6 for an illustration.

Step 5. Choose an edge $e \in \hat{E}^{\circ} \backslash \hat{E} H$ such that one of its head $u$ is even. Denote the other head of $e$ by $v$.

- If $v \in \operatorname{even}(H)$ and $e$ connects different components in $H$, then go to Step 5.1.

- If $v \in \operatorname{even}(H)$ and $u$ and $v$ belong to the same component in $H$, then go to Step 5.2.

- If $v \in$ free $(H)$ and $v=\partial^{-} a$ for some $a \in \hat{B}$, then go to Step 5.3.

- If $v \in$ free $(H)$ and $v \in \partial e^{\prime}$ for some $e^{\prime} \in \hat{M}$, then go to Step 5.4.

- If $v$ is a pseudo-vertex labelled as "saturated," then go to Step 5.5.

If no edge in $\hat{E}^{\circ} \backslash \hat{E}(H)$ satisfies the above conditions, then go to Step 6 .

Step 5.1: Augmentation. Reset $\hat{F}:=M^{\prime} \cup \hat{B}$, where $M^{\prime}:=\hat{M} \triangle\left(P_{u} \cup P_{v} \cup\{e\}\right)$, delete each $T \in \Omega^{\prime}$ with $z\left(\mathcal{L}_{T}\right)=0$ from $\Omega^{\prime}$, and then go to Step 2. See Figure 3 for an illustration.

Step 5.2. Let $C$ be the cycle in $H \cup\{e\}$ and let $U \subseteq V$ be the union of the vertices in $\hat{V} C$. Denote the maximal proper subsets of $U$ belonging to $\Delta$ and $\Upsilon$ by $Y_{1}, \ldots, Y_{k} \in \Delta$ and $W_{1} \ldots, W_{l} \in \Upsilon$. Let $C_{U}$ be an odd cycle in $G_{U}$ obtained by adding even number of edges from each $E_{W_{j}}$ to $C$. For $i=1, \ldots, k$, let $f_{i}^{1}, f_{i}^{2} \in C_{U}$ denote the two edges incident to $Y_{i}$, and let $v_{i}^{1}, v_{i}^{2} \in Y_{i}$ denote the vertices to which $f_{i}^{1}$ and $f_{i}^{2}$ are incident, respectively. If, for some $Y_{i}$, the two vertices $v_{i}^{1}$ and $v_{i}^{2}$ are distinct and $z\left(\mathcal{L}_{Y_{i}^{\prime}}\right)=0$ for the minimal subset $Y_{i}^{\prime} \in \Delta$ of $Y_{i}$ such that $\left\{v_{i}^{1}, v_{i}^{2}\right\} \subseteq Y_{i}^{\prime}$, then go to Step 5.2.1. Otherwise, go to Step 5.2.2.

Step 5.2.1: Deshrinking and augmentation. Delete $Y_{i}^{\prime}$ from $\Delta$ and reset

$$
\hat{M}:= \begin{cases}\left(\hat{M} \triangle P_{Y_{i}}\right) \triangle C & \left(f_{i}^{1}, f_{i}^{2} \in E \backslash M\right), \\ \hat{M} \triangle P_{Y_{i}}^{*} & \text { (otherwise). }\end{cases}
$$

Here, $P_{Y_{i}}^{*}$ denotes the path in $H \cup\{e\}$ from $\hat{S}$ to $Y_{i}$ consisting of odd number of edges. Delete each $T \in \Omega^{\prime}$ with $z\left(\mathcal{L}_{T}\right)=0$ from $\Omega^{\prime}$, and then go to Step 2. See Figure 7 for an illustration.

Step 5.2.2: Shrinking. Without loss of generality, assume that $v_{i}^{1}$ and $v_{i}^{2}$ are identical for $i=$ $1, \ldots, j$, and distinct for $i=j+1, \ldots, k$. For $i=j+1, \ldots, k$, let $Y_{i}^{\prime} \in \Delta$ be the minimal subset of $Y_{i}$ such that $\left\{v_{i}^{1}, v_{i}^{2}\right\} \subseteq Y_{i}^{\prime}$. Let $U^{\prime}=\left(U \backslash\left(Y_{1} \cup \cdots \cup Y_{k}\right)\right) \cup\left\{v_{Y_{1}}, \ldots, v_{Y_{j}}\right\} \cup\left(Y_{j+1}^{\prime} \cup \cdots \cup Y_{k}^{\prime}\right)$. Add $U^{\prime}$ to $\Upsilon$, and define a partition $\mathcal{L}_{U^{\prime}}$ of $U^{\prime}$ by the collection of $\left\{v_{Y_{1}}\right\}, \ldots,\left\{v_{Y_{j}}\right\}, Y_{j+1}^{\prime}, \ldots, Y_{k}^{\prime}$ and singletons of the other vertices in $U^{\prime}$. See Figure 8 for an illustration.

Step 5.3: Augmentation. Reset $\hat{F}:=\left(\hat{M} \triangle P_{v}\right) \cup(\hat{B} \backslash\{a\})$, delete each $T \in \Omega^{\prime}$ with $z\left(\mathcal{L}_{T}\right)=0$ from $\Omega^{\prime}$, and then go to Step 2. See Figure 10 for an illustration.

Step 5.4: Forest extension. Grow $H$ by adding $e$ and $e^{\prime}$. Label $v$ as odd and the other head of $e^{\prime}$ as even. Then, go to Step 3. 

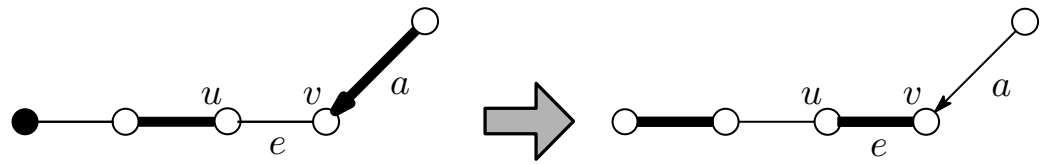

Figure 10: Augmentation in Step 5.3. (Thick edges are in $\hat{F}$ and the black vertex is a source vertex.)

Step 5.5: Augmentation. Reset $\hat{F}:=M^{\prime} \cup \hat{B}$, where $M^{\prime}:=\hat{M} \triangle P_{v}$, and unlabel $v$. Delete each $T \in \Omega^{\prime}$ with $z\left(\mathcal{L}_{T}\right)=0$ and then go to Step 2 .

Step 6. Apply Dual_Update described below, delete each $T \in \Omega^{\prime}$ with $z\left(\mathcal{L}_{T}\right)=0$ from $\Omega^{\prime}$, and then go to Step 3.

Procedure Dual_Update. Define families of vertex subsets of $V$ as follows:

$$
\begin{aligned}
& \Delta_{+}:=\{\text {maximal set in } \Delta, \text { contained in some even vertex }\}, \\
& \Delta_{-}:=\{\text {maximal set in } \Delta, \text { contained in some odd vertex }\}, \\
& \Upsilon_{+}:=\{\text {maximal set in } \Upsilon, \text { contained in some even vertex }\} \\
& \Upsilon_{-}:=\{\text {maximal set in } \Upsilon \text {, contained in some odd vertex }\}
\end{aligned}
$$

Moreover, let

$$
\begin{aligned}
\Delta_{+}^{\prime} & :=\left\{X \subseteq V \mid X \in \Delta, \text { maximal proper subset of some element in } \Upsilon_{+}\right\}, \\
\Delta_{-}^{\prime} & :=\left\{X \subseteq V \mid X \in \Delta, \text { maximal proper subset of some element in } \Upsilon_{-}\right\}, \\
V_{+} & :=\{v \in V \mid\{v\} \in \operatorname{even}(H) \text { or } v \text { is contained in some even vertex }\}, \\
V_{-} & :=\{v \in V \mid\{v\} \in \operatorname{odd}(H) \text { or } v \text { is contained in some odd vertex }\} .
\end{aligned}
$$

Then, update $(y, z)$ by

$$
\begin{aligned}
& y(v):= \begin{cases}y(v)-\epsilon & \left(v \in V_{+}\right), \\
y(v)+\epsilon & \left(v \in V_{-}\right), \\
y(v) & (\text { otherwise }),\end{cases} \\
& z\left(\mathcal{L}_{U}\right):= \begin{cases}z\left(\mathcal{L}_{U}\right)+2 \epsilon & \left(U \in \Upsilon_{+} \cup\left(\Delta_{-}^{\prime} \backslash \Delta_{-}\right)\right), \\
z\left(\mathcal{L}_{U}\right)-2 \epsilon & \left(U \in \Upsilon_{-} \cup\left(\Delta_{+}^{\prime} \backslash \Delta_{+}\right)\right), \\
z\left(\mathcal{L}_{U}\right)+\epsilon & \left(U \in\left(\Delta_{+} \backslash \Delta_{+}^{\prime}\right) \cup\left(\Delta_{-} \cap \Delta_{-}^{\prime}\right)\right), \\
z\left(\mathcal{L}_{U}\right)-\epsilon & \left(U \in\left(\Delta_{+} \cap \Delta_{+}^{\prime}\right) \cup\left(\Delta_{-} \backslash \Delta_{-}^{\prime}\right)\right), \\
z\left(\mathcal{L}_{U}\right) & \text { (otherwise), }\end{cases}
\end{aligned}
$$

where $\epsilon \geq 0$ is the maximum value maintaining (4)-(7). That is, $\epsilon$ is the minimum of the following:

$$
\begin{aligned}
& \epsilon_{1}=\min \left\{y(v) \mid v \in V_{+}\right\} ; \quad \epsilon_{2}=\min \left\{z\left(\mathcal{L}_{U}\right) / 2 \mid U \in \Upsilon_{-} \cup\left(\Delta_{+}^{\prime} \backslash \Delta_{+}\right)\right\} ; \\
& \epsilon_{3}=\min \left\{z\left(\mathcal{L}_{U}\right) \mid U \in\left(\Delta_{+} \cap \Delta_{+}^{\prime}\right) \cup\left(\Delta_{-} \backslash \Delta_{-}^{\prime}\right)\right\} ; \quad \epsilon_{4}=\min \left\{w^{\prime}(e) / 2 \mid e \in \hat{E}, \partial e \subseteq V_{+}\right\} ; \\
& \epsilon_{5}=\min \left\{w^{\prime}(e) \mid e \in \hat{E}, \text { one of } \partial e \text { belongs to } V_{+}, \text {and the other } V \backslash\left(V_{+} \cup V_{-}\right)\right\} ; \\
& \epsilon_{6}=\min \left\{w^{\prime}(e) \mid \partial e \subseteq X \text { for some } X \in \Delta_{+} \cup \Delta_{+}^{\prime}\right\} ; \\
& \epsilon_{7}=\min \left\{w^{\prime}(a) \mid a \in A, \partial^{-} a \in V_{+}, a \notin \gamma\left(\mathcal{L}_{U}\right) \text { for any } U \in \Delta_{+} \cup \Upsilon_{+}\right\} .
\end{aligned}
$$

Then, apply one of the following. 


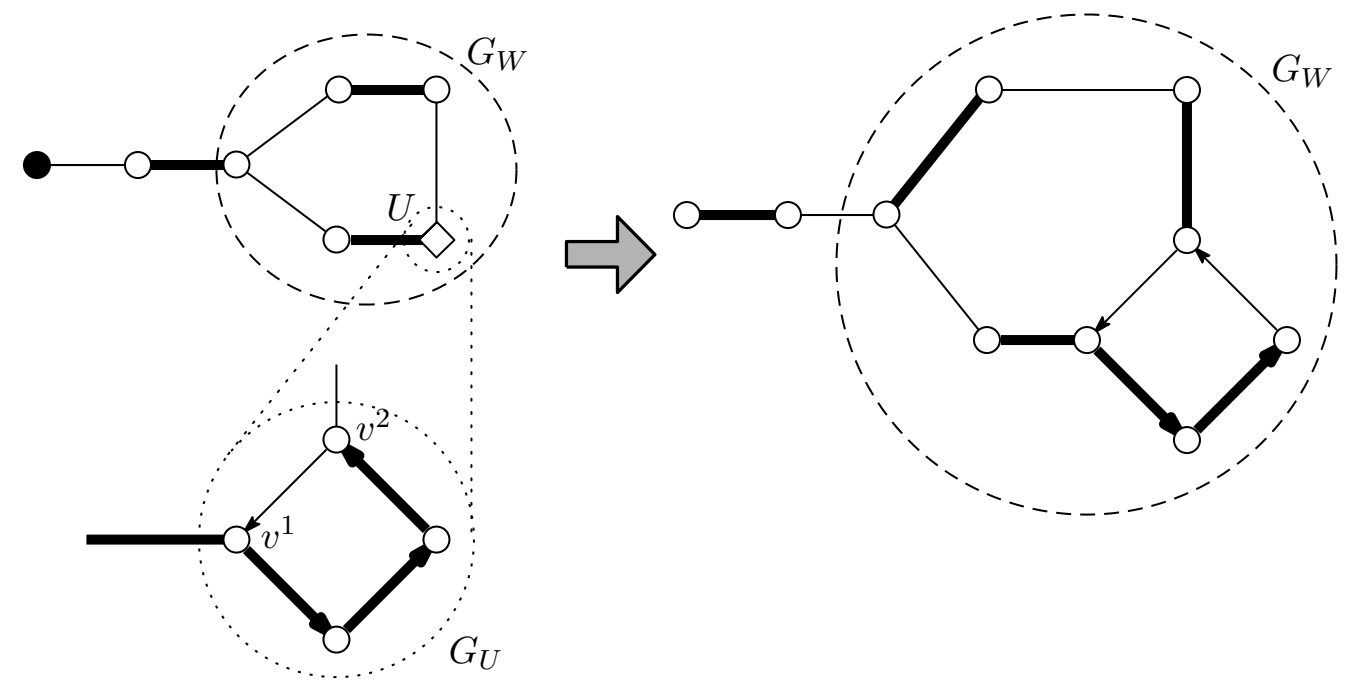

Figure 11: Deshrinking of $U$ and saturation in Case 3.1. (Thick edges are in $\hat{F}$ and the black vertex is a source vertex. The graph inside the dashed ellipse indicates $G_{W}$, and the graph inside the dotted circle indicates $G_{U}$. Both $G_{W}$ and $G_{U}$ are shrunk in $\hat{G}^{\circ}$ before the present Dual_Update. After the present Dual_Update, $U$ is deshrunk whereas $W$ is kept shrunk and labelled as saturated.)

Case $1\left(\epsilon=\epsilon_{1}\right)$ : Termination. Deshrink every sets in $\Omega^{\prime}$ and return $F$.

Case $2\left(\epsilon=\epsilon_{2}\right)$ : Deshrinking. Apply Case 2.1 or 2.2 .

Case $2.1\left(\boldsymbol{\epsilon}=\boldsymbol{z}\left(\mathcal{L}_{U}\right) / 2\right.$ for some $\left.\boldsymbol{U} \in \boldsymbol{\Upsilon}_{-}\right)$: Deshrinking. Delete $U$ with $\epsilon=z\left(\mathcal{L}_{U}\right) / 2$ from $\Upsilon$, and then go to Step 3 .

Case $2.2\left(\epsilon=z\left(\mathcal{L}_{U}\right) / 2\right.$ for some $\left.U \in \Delta_{+}^{\prime} \backslash \Delta_{+}\right)$: Deshrinking. Denote the maximal set in $\Upsilon$ containing $U$ by $W$, and the maximal set in $\Delta$ containing $U$ by $X$. Add $\tilde{U}=W \cup X$ to $\Upsilon$, define a partition $\mathcal{L}_{\tilde{U}}$ of $\tilde{U}$ by $\left(\mathcal{L}_{U} \backslash\{U\}\right) \cup\{X\}$, and set $z\left(\mathcal{L}_{\tilde{U}}\right)=0$. Then, delete $U$ from $\Delta$ and go to Step 3.

Case $3\left(\epsilon=\epsilon_{3}\right)$. Apply Case 3.1 or 3.2 .

Case 3.1 $\left(\epsilon=z\left(\mathcal{L}_{U}\right)\right.$ for some $\left.U \in \Delta_{+} \cap \Delta_{+}^{\prime}\right)$ : Deshrinking and saturation. Denote the set in $\Upsilon_{+}$containing $U$ by $W$, and the pseudo-vertex containing $W$ by $\hat{W}$. Delete $U$ from $\Delta$, reset $\hat{F}:=\left(\hat{M} \triangle P_{\hat{W}}\right) \cup \hat{B}$, and label $\hat{W}$ as "saturated." (Note that $G_{W}^{\circ}$ has a matching forest covering all vertices in $G_{W}^{\circ}$.) Delete each $T \in \Omega^{\prime}$ with $z\left(\mathcal{L}_{T}\right)=0$ from $\Omega^{\prime}$, and then go to Step 2. See Figure 11 for an illustration.

Case $3.2\left(\boldsymbol{\epsilon}=\boldsymbol{z}\left(\mathcal{L}_{U}\right)\right.$ for some $\left.\boldsymbol{U} \in \boldsymbol{\Delta}_{-} \backslash \boldsymbol{\Delta}_{-}^{\prime}\right)$. Let $\hat{U}$ be the vertex in $\hat{V}$ containing $U$, and let $f_{1}, f_{2} \in H$ be the two edges incident to $\hat{U}$. If $f_{1}$ and $f_{2}$ are incident to distinct vertices in $G_{U}$, then apply Case 3.2.1. Otherwise, apply Case 3.2.2.

Case 3.2.1: Deshrinking and augmentation. Reset $\hat{M}:=\hat{M} \triangle P_{\hat{U}}$. Delete $U$ from $\Delta$ and each $T \in \Omega^{\prime}$ with $z\left(\mathcal{L}_{T}\right)=0$ from $\Omega^{\prime}$, and then go to Step 2. See Figure 12 for an illustration.

Case 3.2.2: Deshrinking. Delete $U$ from $\Delta$ and then go to Step 3. See Figure 13 for an illustration. 

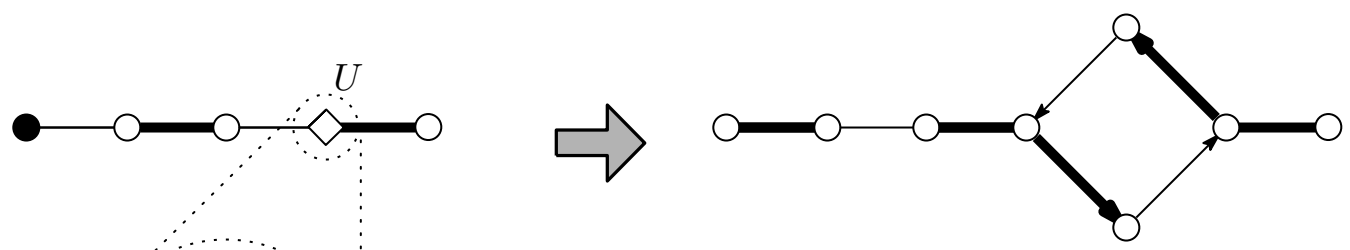

Figure 12: Deshrinking of $U$ and augmentation in Case 3.2.1. (Thick edges are in $\hat{F}$ and the black vertex is a source vertex. The graph inside the dotted circle indicates $G_{U}$.)
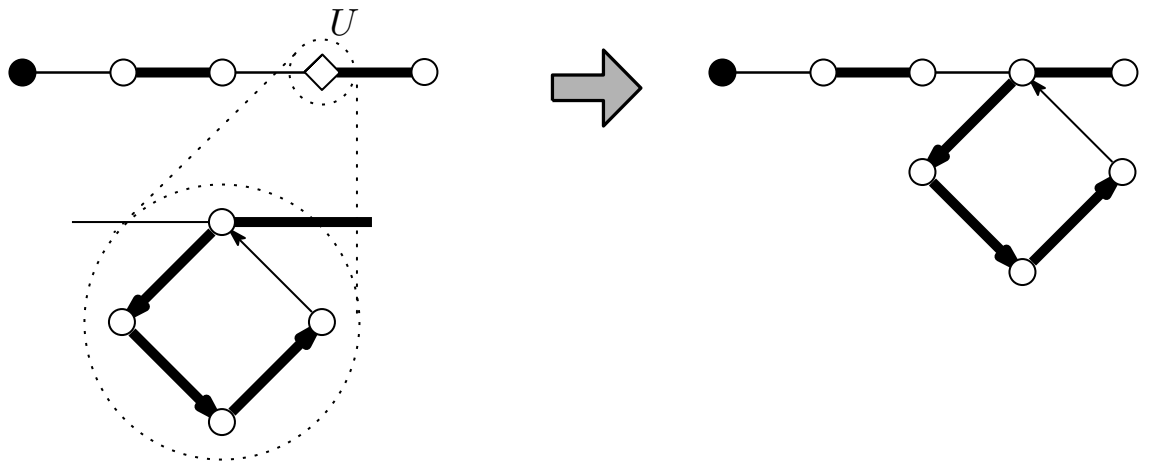

Figure 13: Deshrinking of $U$ in Case 3.2.2. (Thick edges are in $\hat{F}$ and the black vertex is a source vertex. The graph inside the dotted circle indicates $G_{U}$.) 

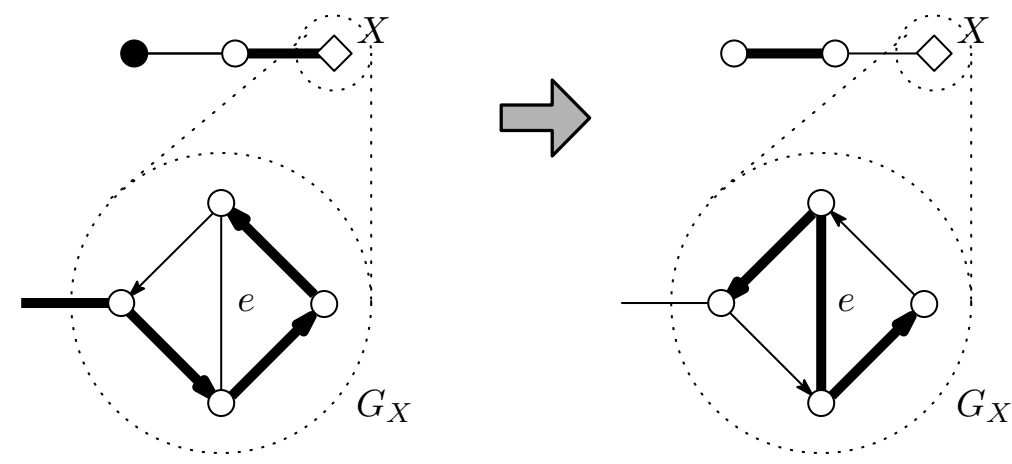

Figure 14: Saturation in Case 6. (Thick edges are in $\hat{F}$ and the black vertex is a source vertex. The graph inside the dotted circle indicates $G_{X}$, and the undirected edge $e \in E$ satisfies that $w^{\prime}(e)=\epsilon$. After the present Dual_Update, $X$ is kept shrunk and labelled as "saturated.")

Case $4\left(\boldsymbol{\epsilon}=\epsilon_{4}\right)$. Go to Step 5. (We can execute Step 5.1 or 5.2.)

Case $5\left(\boldsymbol{\epsilon}=\epsilon_{\mathbf{5}}\right)$. Go to Step 5. (We can execute Step 5.3 or 5.4.)

Case $6\left(\boldsymbol{\epsilon}=\boldsymbol{\epsilon}_{6}\right)$ : Saturation. Let $X \subseteq V$ be an element in $\Delta_{+} \cup \Delta_{+}^{\prime}$ such that contains $e \in E$ with $\epsilon=w^{\prime}(e)$, and let $\hat{X}$ denote the pseudo-vertex in $\hat{G}$ containing $X$. Reset $\hat{M}:=\hat{M} \triangle P_{\hat{X}}$ and label $\hat{X}$ as "saturated." Delete each $T \in \Omega^{\prime}$ with $z\left(\mathcal{L}_{T}\right)=0$ from $\Omega^{\prime}$, and then go to Step 2. See Figures 14 and 15 for an illustration.

Case $7\left(\epsilon=\epsilon_{7}\right)$. Apply Case 7.1 or 7.2 .

Case 7.1 $\left(\epsilon=w^{\prime}(a)\right.$ for some $\left.a \in \hat{A}\right)$. Go to Step 4 .

Case 7.2 $\left(\epsilon=\boldsymbol{w}^{\prime}(\boldsymbol{a})\right.$ for some $\boldsymbol{a} \in \boldsymbol{A}_{\boldsymbol{U}}$ with $\left.\boldsymbol{U} \in \boldsymbol{\Upsilon}_{+}\right)$: Saturation. Reset $\hat{M}:=\hat{M} \triangle P_{U}$ and label $U$ as "saturated." Delete each $T \in \Omega^{\prime}$ with $z\left(\mathcal{L}_{T}\right)=0$ from $\Omega^{\prime}$, and then then go to Step 2. See Figure 16 for an illustration.

\subsection{Validity and complexity}

In this subsection, we verify Algorithm SIMPLE. Our verification is threefold: check if the feasibility is maintained; check if (8) and (10) are maintained; and prove that (9) is achieved in polynomial time.

\subsubsection{Feasibility}

It is obvious that the initial primal and dual solutions defined in Step 1 are feasible. Feasibility of the primal solution all through the algorithm is also clear. We check the dual feasibility conditions (4)(7) after executing Dual_Update.

Condition (6) directly follows from $\epsilon \leq \epsilon_{1}$. Condition (7) also follows from $\epsilon \leq \epsilon_{2}$ and $\epsilon \leq \epsilon_{3}$. Consider Condition (4). If $e \in E$ does not belong to $\gamma\left(\mathcal{L}_{U}\right)$ for any $U \in \Omega^{\prime}$, then $w^{\prime}(e)$ decreases in the following two cases.

- $\boldsymbol{\partial e} \subseteq \boldsymbol{V}_{+}$. In this case, $w^{\prime}(e)$ decreases by $2 \epsilon$. We have that $w^{\prime}(e) \geq 0$ is maintained since $\epsilon \leq \epsilon_{4}$. 

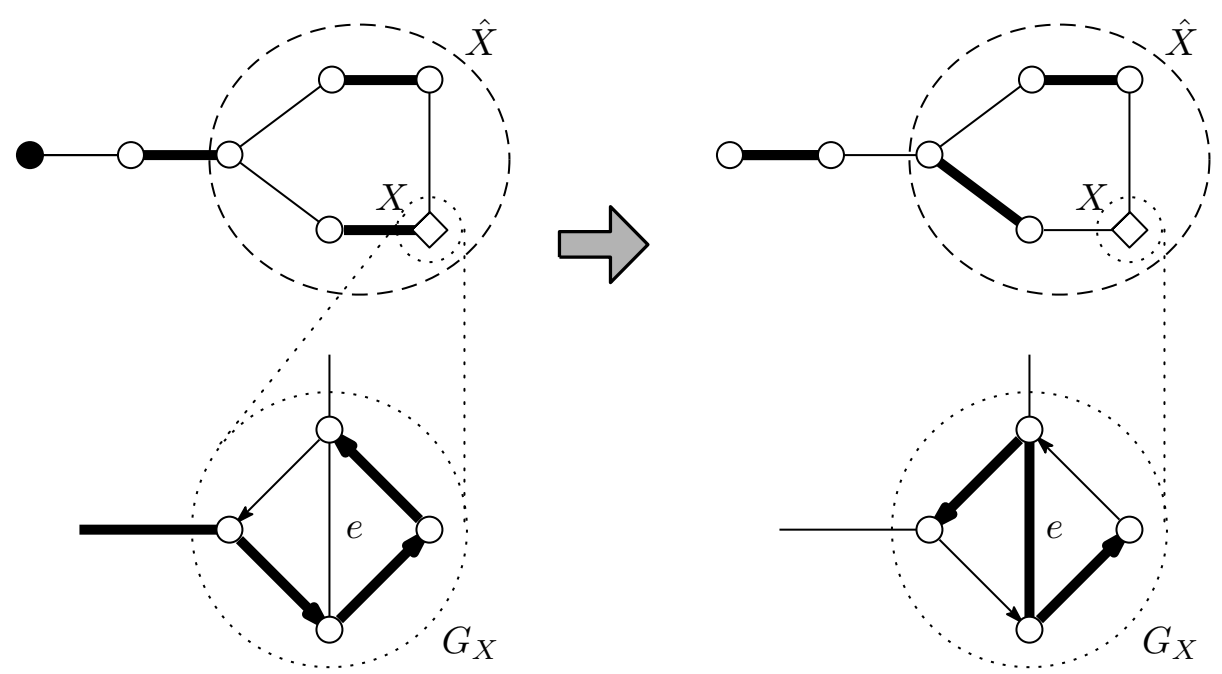

Figure 15: Saturation in Case 6. (Thick edges are in $\hat{F}$ and the black vertex is a source vertex. The graph inside the dashed ellipse a subgraph shrunk into the pseudo-vertex $\hat{X}$, and that inside the dotted circle indicates $G_{X}$. The edge $e \in E$ satisfies that $w^{\prime}(e)=\epsilon$. Both $G_{\hat{X}}$ and $G_{X}$ are shrunk in $\hat{G}^{\circ}$. After the present Dual_Update, $\hat{X}$ and $X$ are kept shrunk and $\hat{X}$ is labelled as "saturated.")
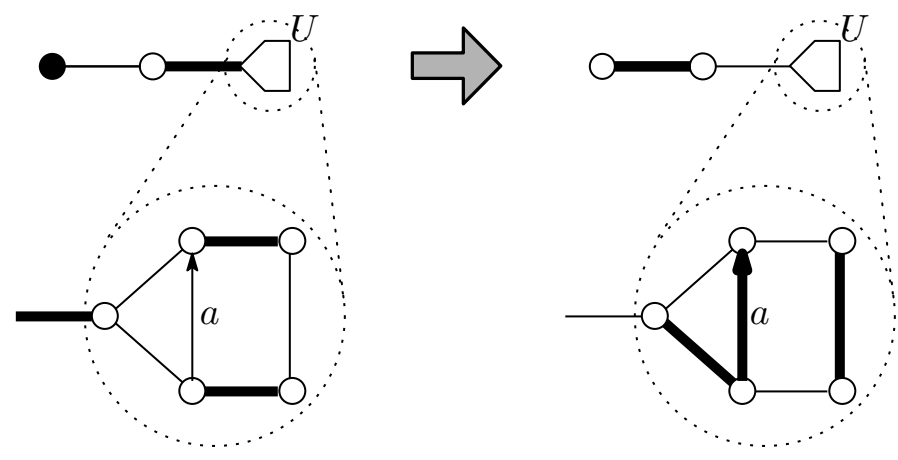

Figure 16: Saturation in Case 7.2. (Thick edges are in $\hat{F}$ and the black vertex is a source vertex. The graph inside the dotted circle indicates $G_{U}$. After the present Dual_Update, $U$ is kept shrunk and labelled as "saturated.") 
- $\left|\partial e \cap V_{+}\right|=1$ and $\left|\partial e \cap V \backslash\left(V_{+} \cup V_{-}\right)\right|=1$. In this case, $w^{\prime}(e)$ decreases by $\epsilon$ and $w^{\prime}(e) \geq 0$ directly follows from $\epsilon \leq \epsilon_{5}$.

If $e \in E$ belongs to $\gamma\left(\mathcal{L}_{U}\right)$ for some $U \in \Omega^{\prime}$, we have the following five cases.

- $e \in \gamma\left(\mathcal{L}_{U}\right)$ for some $\boldsymbol{U} \in \boldsymbol{r}_{+}$. Since $y$ decreases by $\epsilon$ at both endpoints of $e$ and $z\left(\mathcal{L}_{U}\right)$ increases by $2 \epsilon$, the only possibility for $w^{\prime}(e)$ to decrease is that $e$ also belongs to $\gamma\left(\mathcal{L}_{X}\right)$ for some $X \in \Delta_{+}^{\prime}$ contained in $U$. In such a case, $w^{\prime}(e)$ decreases by $\epsilon$ and $w^{\prime}(e) \geq 0$ is maintained since $\epsilon \leq \epsilon_{6}$.

- $e \in \gamma\left(\mathcal{L}_{U}\right)$ for some $\boldsymbol{U} \in \boldsymbol{\Upsilon}_{-}$. In this case, $y$ increases by $\epsilon$ at both endpoints of $e$ and $z\left(\mathcal{L}_{U}\right)$ decreases by $2 \epsilon$. Hence, $w^{\prime}(e)$ does not change if $e \notin \gamma\left(\mathcal{L}_{X}\right)$ for any $X \in \Delta_{-}^{\prime}$ contained in $U$, and increases by $\epsilon$ if $e \in \gamma\left(\mathcal{L}_{X}\right)$ for some $X \in \Delta_{-}^{\prime}$ contained in $U$.

- $e \in \gamma\left(\mathcal{L}_{U}\right)$ for some $\boldsymbol{U} \in \boldsymbol{\Delta}_{+} \backslash \boldsymbol{\Delta}_{+}^{\prime}$. In this case, $w^{\prime}(e)$ decreases by $\epsilon$ since $y$ decreases by $\epsilon$ at both endpoints of $e$ and $z\left(\mathcal{L}_{U}\right)$ increases by $\epsilon$. Here, $w^{\prime}(e) \geq 0$ follows from $\epsilon \leq \epsilon_{6}$.

- $e \in \gamma\left(\mathcal{L}_{\boldsymbol{U}}\right)$ for some $\boldsymbol{U} \in \boldsymbol{\Delta}_{-} \backslash \boldsymbol{\Delta}_{-}^{\prime}$. In this case, $w^{\prime}(e)$ increases by $\epsilon$ since $y$ increases at both endpoints of $e$ and $z\left(\mathcal{L}_{U}\right)$ decreases by $\epsilon$.

- $e \in \gamma\left(\mathcal{L}_{U}\right)$ for some $\boldsymbol{U}$, not in $\boldsymbol{H}$. In this case, $w^{\prime}(e)$ does not change.

Finally, consider Condition (5). If $a \in A$ does not belong to $\gamma\left(\mathcal{L}_{U}\right)$ for $U \in \Omega^{\prime}, w^{\prime}(a) \geq 0$ follows from $\epsilon \leq \epsilon_{7}$. If $a \in A$ belongs to $\gamma\left(\mathcal{L}_{U}\right)$ of some $U \in \Omega^{\prime}$, We have the following five cases.

- $\boldsymbol{a} \in \gamma\left(\mathcal{L}_{U}\right)$ for some $\boldsymbol{U} \in \boldsymbol{\Upsilon}_{+}$. In this case, $a$ also belongs to $\gamma\left(\mathcal{L}_{X}\right)$ for some $X \in \Delta_{+}^{\prime}$ contained in $U$. The dual variables $y\left(\partial^{-} a\right), z\left(\mathcal{L}_{U}\right)$ and $z\left(\mathcal{L}_{X}\right)$ change by $-\epsilon, 2 \epsilon$ and $-\epsilon$, respectively, and hence $w^{\prime}(a)$ does not change.

- $\boldsymbol{a} \in \gamma\left(\mathcal{L}_{\boldsymbol{U}}\right)$ for some $\boldsymbol{U} \in \boldsymbol{\Upsilon}_{-}$. In this case, $a$ also belongs to $\gamma\left(\mathcal{L}_{X}\right)$ for some $X \in \Delta_{-}^{\prime}$ contained in $U$. The dual variables $y\left(\partial^{-} a\right), z\left(\mathcal{L}_{U}\right)$ and $z\left(\mathcal{L}_{X}\right)$ change by $\epsilon,-2 \epsilon$ and $\epsilon$, respectively, and hence $w^{\prime}(a)$ does not change.

- $\boldsymbol{a} \in \gamma\left(\mathcal{L}_{\boldsymbol{U}}\right)$ for some $\boldsymbol{U} \in \boldsymbol{\Delta}_{+} \backslash \boldsymbol{\Delta}_{+}^{\prime}$. In this case, $w^{\prime}(a)$ does not change since $y\left(\partial^{-} a\right)$ decreases by $\epsilon$ and $z\left(\mathcal{L}_{U}\right)$ increases by $\epsilon$.

- $\boldsymbol{a} \in \gamma\left(\mathcal{L}_{\boldsymbol{U}}\right)$ for some $\boldsymbol{U} \in \boldsymbol{\Delta}_{-} \backslash \boldsymbol{\Delta}_{-}^{\prime}$. In this case, $w^{\prime}(a)$ does not change since $y\left(\partial^{-} a\right)$ increases by $\epsilon$ and $z\left(\mathcal{L}_{U}\right)$ decreases by $\epsilon$.

- $\boldsymbol{a} \in \gamma\left(\mathcal{L}_{\boldsymbol{U}}\right)$ for some $\boldsymbol{U}$, not in $\boldsymbol{H}$. In this case, $w^{\prime}(a)$ does not change.

\subsubsection{Complementary slackness conditions}

Consider condition (8). Since the edges in $F$ are picked from $G^{\circ}$, it suffices to check that (8) is maintained in the Dual_Update. For each edge $e \in \hat{M}$ not shrunk, $w^{\prime}(e)$ does not change. Also, it can be easily verified that $w^{\prime}(e)$ does not change for each edge $e$ which has belonged to a shrunk source component or a shrunk odd cycle.

For condition (10). it is not difficult to see that we can deshrink each set $U \in \Omega^{\prime}$ with $z\left(\mathcal{L}_{U}\right)>0$ so that $x(\gamma(\mathcal{L}))=\lfloor|\cup \mathcal{L}|-|\mathcal{L}| / 2\rfloor$. 


\subsubsection{Complexity}

First, we show that $\epsilon>0$ in a Dual_Update, the proof of which implies time complexity of Algorithm SIMPLE.

Proposition 10. In the procedure Dual_Update, it holds that $\epsilon>0$.

Proof. $\left(\epsilon_{1}>0\right)$. If $\epsilon_{1}=0$, then we have that $S=\emptyset$ and Algorithm SIMPLE should have terminated.

$\left(\epsilon_{2}, \epsilon_{3}>0\right)$. A vertex set shrunk after the latest augmentation or saturation is contained in an even vertex in $\hat{G}$. Hence, for each $U \in \Delta_{-} \cup \Upsilon_{-}$, we have that $U$ was shrunk before the latest augmentation or saturation, at which $z\left(\mathcal{L}_{U}\right)>0$. Also, for $U \in \Delta_{+}^{\prime}$, we have that $z\left(\mathcal{L}_{U}\right)>0$ by the bifurcation rule between Steps 5.2.1 and 5.2.2. If $z\left(\mathcal{L}_{U}\right)$ hits 0 for such $U$, then $U$ is deshrunk.

$\left(\boldsymbol{\epsilon}_{\mathbf{4}}>\mathbf{0}\right)$. If there exists $e \in \hat{E}$ such that $w^{\prime}(e)=0$ and $\partial e \subseteq \hat{V}_{+}$, then we do not execute Dual_Update but Step 5.1 or 5.2 .

$\left(\boldsymbol{\epsilon}_{\mathbf{5}}>\mathbf{0}\right)$. If $w^{\prime}(e)=0$ for $e \in \hat{E}$ such that one of $\partial e$ belongs to $\hat{V}_{+}$and the other in $\hat{V} \backslash\left(\hat{V}_{+} \cup \hat{V}_{-}\right)$, then we do not execute Dual_Update but Step 5.3, 5.4 or 5.5.

$\left(\boldsymbol{\epsilon}_{\mathbf{6}}>\mathbf{0}\right)$. Suppose $w^{\prime}(e)=0$ for $e \in E$ such that $\partial e \subseteq X$ for some $X \in \Delta_{+} \cup \Delta_{+}^{\prime}$. Denote the minimal set in $\Delta$ containing $\partial e$ by $X_{e}$. When $X_{e}$ is added to $\Delta$, we have that $w^{\prime}(e)>0$ by the bifurcation rule between Steps 4.2.1 and 4.2.2. If $w^{\prime}(e)$ hit zero for such $e$, then we should have executed saturation of the pseudo-vertex containing $e$.

$\left(\boldsymbol{\epsilon}_{\mathbf{7}}>\mathbf{0}\right)$. If $w^{\prime}(a)=0$ for $a \in A$ attaining $\epsilon_{7}$, then we execute Step 4 or saturation of the pseudovertex containing $a$.

We now discuss the time complexity of Algorithm SIMPLE. The bottleneck part is Dual_Update. It follows from the proof for Proposition 10 that Dual_Update is executed $\mathrm{O}(n)$ times between consecutive augmentations or saturations. Since augmentations and saturations collectively happen at most $n$ times and Dual_Update takes $\mathrm{O}(m)$ time for determining $\epsilon$, the total complexity is $\mathrm{O}\left(n^{2} m\right)$.

Theorem 11. Algorithm SIMPLE finds a maximum-weight matching forest in $\mathrm{O}\left(n^{2} m\right)$ time.

\subsection{Remarks}

We close this section by noting a property of the matching forests maintained in Algorithm SIMPLE: a matching forest appearing at any stage of Algorithm SIMPLE has the maximum weight among all matching forests with the same root-size.

Theorem 12. Let $F$ be a matching forest which we have at any stage of Algorithm SIMPLE. (Deshrink each element in $\Omega^{\prime}$, if $\Omega \neq \emptyset$.) Then, it holds that $w(F) \geq w\left(F^{\prime}\right)$ for any matching forest $F^{\prime}$ with $\left|R\left(F^{\prime}\right)\right|=|R(F)|$. 
Proof. Let $F$ be a matching forest obtained at an arbitrary stage of Algorithm SIMPLE and let $(y, z)$ be the dual solution at that stage. For any matching forest $F^{\prime}$, it holds that

$$
w\left(F^{\prime}\right) \leq y\left(V \backslash R\left(F^{\prime}\right)\right)+\sum_{\mathcal{L} \in \Lambda} z(\mathcal{L})|\gamma(\mathcal{L}) \cap F| \leq y\left(V \backslash R\left(F^{\prime}\right)\right)+\sum_{\mathcal{L} \in \Lambda} z(\mathcal{L})\lfloor|\cup \mathcal{L}|-|\mathcal{L} / 2|\rfloor .
$$

Especially for the matching forest $F$, conditions (8) and (10) imply that

$$
w(F)=y(V \backslash R(F))+\sum_{\mathcal{L} \in \Lambda} z(\mathcal{L})\lfloor|\cup \mathcal{L}|-|\mathcal{L} / 2|\rfloor .
$$

Here, for $v \in R(F)$, we have that $y(v) \leq y\left(v^{\prime}\right)$ for every $v^{\prime} \in V$ since the values of $y \in \mathbf{R}^{V}$ are identical in Step 1 and $y(v)$ is decreased at each Dual_Update for $v \in R(F)$. Thus, $y(V \backslash R(F)) \geq$ $y\left(V \backslash R\left(F^{\prime}\right)\right)$ if $|R(F)|=\left|R\left(F^{\prime}\right)\right|$. Therefore, $w(F) \geq w\left(F^{\prime}\right)$ follows.

\section{A faster algorithm}

In this section, we present an $\mathrm{O}\left(n^{3}\right)$ algorithm for the weighted matching forest problem by incorporating Gabow's technique for weighted matching [13] into Giles' weighted matching forest algorithm [15]. The difference from the algorithm in $\S 3$ is that we do not maintain the equality subgraph $G^{\circ}$ explicitly. Instead, we keep the following.

- For each pair $Y, Z$ of disjoint sets in $\Omega$, we keep an edge $e_{Y Z} \in E$ connecting $Y$ and $Z$ and minimizing $w^{\prime}$. We keep $e_{Y Z}$ as lists: for each $Y \in \Omega$, we have a list containing the $e_{Y Z}$. Moreover, for each $Y \in \Omega$, we keep an edge $e_{Y}$ with $e_{Y}=e_{Y Z}$ for some $Z \in \Omega$ contained in an even (pseudo-)vertex in $H$ and with $w^{\prime}\left(e_{Y Z}\right)$ minimal. Similarly, for each pair $Y, Z$ of disjoint sets in $\Omega$, we keep an $\operatorname{arc} a_{Y Z} \in A$ from $Y$ to $Z$ minimizing $w^{\prime}$. We keep $a_{Y Z}$ as lists: for each $Z \in \Omega$, we have a list containing the $a_{Y Z}$. Moreover, for each $Z \in \Omega$, we keep an arc $a_{Z}$ with $a_{Z}=a_{Y Z}$ for some $Y \in \Omega$ and with $w^{\prime}\left(a_{Y Z}\right)$ minimal.

- For each $X \in \Delta$, we keep an edge $f_{X} \in E_{X}$ minimizing $w^{\prime}$. Also, we associate a graph $G_{X}^{\prime}$, which is initially the directed cycle shrunk when $X$ is added to $\Delta$.

- For each $U \in \Upsilon$, we keep an $\operatorname{arc} b_{U} \in A_{U}$ minimizing $w^{\prime}$. We also associate graph $G_{U}^{\prime}$, which is initially the odd undirected cycle shrunk when $U$ is added to $\Upsilon$.

The algorithm is described below.

\section{Algorithm FAST}

Initialization. Set $F:=\emptyset, y(v):=\max \{\max \{w(e) / 2 \mid e \in E\}, \max \{w(a) \mid a \in A\}\}$ for every $v \in V, \Delta:=\emptyset$ and $\Upsilon:=\emptyset$. (Hence $\Omega=\{\{v\} \mid v \in V\}, \hat{F}=\emptyset$ and $z$ is void.) Moreover, set $H=\emptyset$. The $e_{Y Z}, e_{Y}, a_{Y Z}$ and $a_{Z}$ and are set easily.

Iteration. Reset $(y, z)$ as described in Procedure Dual_Update in Algorithm SIMPLE. After that, at least one of the following cases applies.

Case $1\left(w^{\prime}\left(a_{U}\right)=0\right.$ for some maximal set $U \in \Omega$ in even $\left.(\boldsymbol{H})\right)$. Denote $B^{\prime}:=\hat{B} \cup\left\{a_{U}\right\}$. 
Case $1.1\left(B^{\prime}\right.$ is a branching): Augmentation. Let $M^{\prime}:=M \triangle P_{U}$. Reset $\hat{M}:=M^{\prime}, \hat{B}:=B^{\prime}$ and $H:=M^{\prime}$, and update the $e_{Y}$.

Case $1.2\left(B^{\prime}\right.$ contains a directed cycle). Let $D$ be the directed cycle in $B^{\prime}$ and $X \subseteq V$ be the union of the vertices in $D$. Let $W_{1}, \ldots, W_{l}$ be the maximal proper subsets of $X$ belonging to $\Upsilon$. If, for some $i \in\{1, \ldots, l\}, \hat{A} D$ contains a pair of arcs $f^{+} \in \delta^{+} W_{i}$ and $f^{-} \in \delta^{-} W_{i}$ such that $\partial^{+} f^{+}$and $\partial^{-} f^{-}$belong to distinct vertices in $G_{W_{i}}^{\prime}$, apply Case 1.2.1. Otherwise, apply Case 1.2.2.

Case 1.2.1: Augmentation. Reset $\hat{F}:=M^{\prime} \cup B^{\prime}$, where $M^{\prime}:=\hat{M} \triangle P_{U}$ and $B^{\prime}:=\hat{B} \cup\left\{a_{U}\right\}$, and $H:=M^{\prime}$, and update the $e_{Y}$.

Case 1.2.2: Shrinking. For each $i=1, \ldots, l$ let $v_{W_{i}} \in V_{W_{i}}$ denote the unique vertex in $G_{W_{i}}$ to which arcs in $\hat{A} D$ are incident. Let $X^{\prime}=\left(X \backslash\left(W_{1} \cup \cdots \cup W_{l}\right)\right) \cup\left\{v_{W_{i}}\right\} \cup \cdots \cup\left\{v_{W_{l}}\right\}$ and add $X^{\prime}$ to $\Delta$. Let $\mathcal{L}_{X^{\prime}}=\left\{X^{\prime}\right\}$, set $z\left(\mathcal{L}_{X^{\prime}}\right):=0$ and determine $f_{X}$. Then, update the $e_{Y Z}, e_{Y}$, $a_{Y Z}$ and $a_{Z}$.

Case $2\left(w^{\prime}\left(e_{U}\right)=0\right.$ for some maximum set $\boldsymbol{U} \in \Omega$ in even $\left.(\boldsymbol{H})\right)$. Denote the other endpoint of $e_{U}$ by $W$, which also belongs to even $(H)$. Apply Case 2.1 or 2.2.

Case $2.1\left(\boldsymbol{P}_{\boldsymbol{U}}\right.$ and $\boldsymbol{P}_{\boldsymbol{W}}$ are disjoint): Augmentation. Reset $\hat{M}:=M^{\prime}=\hat{M} \triangle\left(P_{U} \cup\{e\} \cup P_{W}\right)$, $H:=M^{\prime}$, and update the $e_{Y}$.

Case $2.2\left(\boldsymbol{P}_{\boldsymbol{U}}\right.$ and $\boldsymbol{P}_{\boldsymbol{W}}$ intersect). Let $C$ be the cycle in $H \cup\left\{e_{U}\right\}$ and $U \subseteq V$ be the union of the vertices in $C$. Denote the maximal proper subsets of $U$ belonging to $\Delta$ and $\Upsilon$ by $Y_{1}, \ldots, Y_{k} \in \Delta$ and $W_{1} \ldots, W_{l} \in \Upsilon$. Let $C_{U}$ be an odd cycle in $G_{U}$ obtained by adding even number of edges from each $G_{W_{j}}^{\prime}$ to $C$. For $i=1, \ldots, k$, let $f_{i}^{1}, f_{i}^{2} \in C_{U}$ denote the two edges incident to $Y_{i}$, and let $v_{i}^{1}, v_{i}^{2} \in Y_{i}$ denote the vertices to which $f_{i}^{1}$ and $f_{i}^{2}$ are incident, respectively. If, for some $Y_{i}$, the two vertices $v_{i}^{1}$ and $v_{i}^{2}$ are distinct and $z\left(\mathcal{L}_{Y_{i}^{\prime}}\right)=0$ for the minimal subset $Y_{i}^{\prime} \in \Delta$ of $Y_{i}$ such that $\left\{v_{i}^{1}, v_{i}^{2}\right\} \subseteq Y_{i}^{\prime}$, then apply Case 2.2.1. Otherwise, go to apply Case 2.2.2.

Case 2.2.1: Deshrinking and augmentation. Delete $Y_{i}^{\prime}$ from $\Delta$ and reset

$$
\hat{M}:= \begin{cases}\left(\hat{M} \triangle P_{Y_{i}}\right) \triangle C & \left(f_{i}^{1}, f_{i}^{2} \in E \backslash M\right), \\ \hat{M} \triangle P_{Y_{i}}^{*} & \text { (otherwise) }\end{cases}
$$

Here, $P_{Y_{i}}^{*}$ denotes the path in $H \cup\{e\}$ from $\hat{S}$ to $Y_{i}$ consisting of odd number of edges. Reset $H:=M^{\prime}$, and update the $e_{Y Z}, e_{Y}, a_{Y Z}$ and $a_{Z}$.

Case 2.2.2: Shrinking. Without loss of generality, assume that $v_{i}^{1}$ and $v_{i}^{2}$ are identical for $i=$ $1, \ldots, j$, and distinct for $i=j+1, \ldots, k$. For $i=j+1, \ldots, k$, let $Y_{i}^{\prime} \in \Delta$ be the minimal subset of $Y_{i}$ such that $\left\{v_{i}^{1}, v_{i}^{2}\right\} \subseteq Y_{i}^{\prime}$. Let $U^{\prime}=\left(U \backslash\left(Y_{1} \cup \cdots \cup Y_{k}\right)\right) \cup\left\{v_{Y_{1}}, \ldots, v_{Y_{j}}\right\} \cup$ $\left(Y_{j+1}^{\prime} \cup \cdots Y_{k}^{\prime}\right)$. We now add $U^{\prime}$ to $\Upsilon$, and define a partition $\mathcal{L}_{U^{\prime}}$ of $U^{\prime}$ by the collection of $\left\{v_{Y_{1}}\right\}, \ldots,\left\{v_{Y_{j}}\right\}, Y_{j+1}^{\prime}, \ldots, Y_{k}^{\prime}$ and singletons of the other vertices in $U^{\prime}$. Set $z\left(\mathcal{L}_{U^{\prime}}\right)=0$, determine $b_{U^{\prime}}$ and update the $e_{Y Z}, e_{Y}, a_{Y Z}$ and $a_{Z}$.

Case $3\left(\boldsymbol{w}^{\prime}\left(e_{\boldsymbol{U}}\right)=\mathbf{0}\right.$ for some $\boldsymbol{U} \in$ free $\left.(\boldsymbol{H})\right)$. Apply Case 3.1 or 3.2.

Case 3.1 ( $U \in \partial^{-} a$ for some $a \in \hat{B}$ or $U$ saturated): Augmentation. Denote the endpoint of $e_{U}$ other than $U$ by $W$. Reset $\hat{M}:=M^{\prime}=\left(\hat{M} \triangle P_{W}\right) \cup\left\{e_{U}\right\}, \hat{B}:=\hat{B} \backslash\{a\}, H:=M^{\prime}$, and update the $e_{Y}$. If $U$ is a saturated pseudo-vertex, unlabel $U$. 
Case $3.2\left(\boldsymbol{U} \in \hat{\boldsymbol{V}} \backslash \boldsymbol{\partial}^{-} \hat{\boldsymbol{B}}\right)$ : Forest extension. Add $e_{U}$ to $H$ and update the $e_{Y}$.

Case $4\left(z\left(\mathcal{L}_{U}\right)=0\right.$ for some $\left.U \in \Upsilon_{-}\right)$: Deshrinking. Delete $U$ from $\Upsilon$. Let $u \in \hat{V}$ be the vertex covered by $H \backslash \hat{M}$ and $v \in \hat{V}$ be the one covered by $\hat{M}$. Let $P$ be the even-length $u-v$ path in $G_{U}^{\prime}$ and $N$ be the matching in $G_{U}^{\prime}$ covering all vertices in the odd cycle other than $v$. Reset $H:=H \cup P \cup N$ and $\hat{M}:=\hat{M} \cup N$, and update the $e_{Y Z}, e_{Y}, a_{Y Z}$ and $a_{Z}$.

Case $5\left(z\left(\mathcal{L}_{U}\right)=\mathbf{0}\right.$ for some $\left.U \in \Delta_{+}^{\prime} \cup\left(\Delta_{-} \backslash \Delta_{-}^{\prime}\right)\right)$ : Deshrinking. Apply Case 5.1, 5.2 or 5.3 .

Case $5.1\left(\boldsymbol{U} \in \boldsymbol{\Delta}_{-} \backslash \boldsymbol{\Delta}_{-}^{\prime}\right)$. Let $u \in V_{U}$ be the vertex covered by $H \backslash \hat{M}$ and $v$ be the one covered by $\hat{M}$. If $u$ and $v$ are distinct, then apply Case 5.1.1. Otherwise, apply Case 5.1.2.

Case 5.1.1: Deshrinking and augmentation. Delete $U$ from $\Delta$, reset $\hat{M}:=M^{\prime}=\hat{M} \triangle P_{U}$, $H:=M^{\prime}$, and update the $e_{Y Z}, e_{Y}, a_{Y Z}$ and $a_{Z}$.

Case 5.1.2: Deshrinking. Delete $U$ from $\Delta$, and update the $e_{Y Z}, e_{Y}, a_{Y Z}$ and $a_{Z}$.

Case $5.2\left(U \in \boldsymbol{\Delta}_{+}^{\prime} \backslash \boldsymbol{\Delta}_{+}\right)$: Deshrinking. Denote the set in $\Upsilon_{+}$containing $U$ by $W$, and the maximal set in $\Delta$ containing $U$ by $X$. Delete $U$ from $\Delta$, add $\tilde{U}=W \cup X$ to $\Upsilon$, define a partition $\mathcal{L}_{\tilde{U}}$ of $\tilde{U}$ by $\left(\mathcal{L}_{U} \backslash\{U\}\right) \cup\{X\}$, and set $z\left(\mathcal{L}_{\tilde{U}}\right)=0$. Then, update the $e_{Y Z}, e_{Y}, a_{Y Z}$ and $a_{Z}$.

Case $5.3\left(U \in \Delta_{+} \cap \Delta_{+}^{\prime}\right)$ : Deshrinking and saturation. Denote the set in $\Upsilon_{+}$containing $U$ by $W$, and the pseudo-vertex containing $W$ by $\hat{W}$. Delete $U$ from $\Delta$, reset $\hat{M}:=M^{\prime}=$ $\left(\hat{M} \triangle P_{\hat{W}}\right)$, and label $\hat{W}$ as "saturated." Reset $H:=M^{\prime}$ and update the $e_{Y Z}, e_{Y}, a_{Y Z}$ and $a_{Z} \cdot$

Case $6\left(\boldsymbol{w}^{\prime}\left(\boldsymbol{f}_{\boldsymbol{X}}\right)=\mathbf{0}\right.$ for some $\left.\boldsymbol{X} \in \boldsymbol{\Delta}_{+} \cup \boldsymbol{\Delta}_{+}^{\prime}\right)$ : Saturation. Let $\hat{X}$ denote the pseudo-vertex in $\hat{G}$ containing $X$. Add $f_{X}$ to $G_{X}^{\prime}$. Reset $\hat{M}:=M^{\prime}=\hat{M} \triangle P_{\hat{X}}$ and label $\hat{X}$ as "saturated." Reset $H:=M^{\prime}$ and update the $e_{Y}$.

Case $7\left(\boldsymbol{w}^{\prime}\left(\boldsymbol{b}_{\boldsymbol{U}}\right)=\mathbf{0}\right.$ for some $\left.\boldsymbol{U} \in \boldsymbol{\Upsilon}_{+}\right)$: Saturation. Let $\hat{U}$ denote the pseudo-vertex containing $U$. Add $b_{U}$ to $G_{U}^{\prime}$. Reset $\hat{M}:=M^{\prime}=M \triangle P_{\hat{U}}$ and label $\hat{U}$ as "saturated." Reset $H:=M^{\prime}$ and update the $e_{Y}$.

The validity of Algorithm FAST can be verified just as we did for Algorithm SIMPLE. Let us mention here the complexity. In Algorithm FAST, Procedure Dual_Update can be executed in $\mathrm{O}(n)$ time, by scanning the $e_{Y}, a_{Z}, f_{X}$ and $b_{U}$, together with the $z\left(\mathcal{L}_{U}\right)$, instead of scanning all edges in $E \cup A$. Updating the lists after shrinking, deshrinking or forest extension takes $\mathrm{O}(n)$ time and updating after augmentation and saturation takes $\mathrm{O}\left(n^{2}\right)$ time. Thus, the total time complexity $\mathrm{O}\left(n^{3}\right)$.

Theorem 13. Algorithm FAST finds a maximum-weight matching forest in $\mathrm{O}\left(n^{3}\right)$ time.

We remark that we do not incorporate the branching delta-matroid $\hat{F}_{B}^{\circ}$, which is used in Algorithm SIMPLE. This is because we need to decompose the equality subgraph $\left(\hat{V}, \hat{A}^{\circ}\right)$ into strong components in order to determine whether $R(\hat{B}) \backslash\{v\} \in \hat{\mathcal{F}}_{B}^{\circ}$, which takes $\mathrm{O}(m)$ time. 


\section{Acknowledgements}

The author is thankful to Satoru Iwata for valuable comments on this research and the simple proof for the matching forest delta-matroid. The author also thanks Yusuke Kobayashi for discussions on simultaneous delta-matroids.

\section{References}

[1] A. Bouchet: Greedy algorithm and symmetric matroids, Mathematical Programming, 38 (1987), 147-159.

[2] A. Bouchet: Matchings and $\Delta$-matroids, Discrete Applied Mathematics, 24 (1989), 55-62.

[3] R. Chandrasekaran and S.N. Kabadi: Pseudomatroids, Discrete Mathematics, 71 (1988), 205217.

[4] W.H. Cunningham and A.B. Marsh, III: A primal algorithm for optimum matching, Mathematical Programming Study, 8 (1978), 50-72.

[5] A.W.M. Dress and T. Havel: Some combinatorial properties of discriminants in metric vector spaces, Advances in Mathematics, 62 (1986), 285-312.

[6] A.W.M. Dress and W. Wenzel: A greedy-algorithm characterization of valuated $\Delta$-matroids, Applied Mathematics Letters, 4 (1991), 55-58.

[7] J. Edmonds: Maximum matching and a polyhedron with 0,1-vertices, Journal of Research National Bureau of Standards, Section B, 69 (1965), 125-130.

[8] J. Edmonds: Paths, trees, and flowers, Canadian Journal of Mathematics, 17 (1965), 449-467.

[9] J. Edmonds: Optimum branchings, Journal of Research National Bureau of Standards, Section B, 71 (1967), 233-240.

[10] J. Edmonds and R. Giles: A min-max relation for submodular functions on graphs, Annals of Discrete Mathematics, 1 (1977), 185-204.

[11] A. Frank: Covering branchings, Acta Scientiarum Mathematicarum [Szeged], 41 (1979), 77-81.

[12] A. Frank and E. Tardos: Generalized polymatroids and submodular flows, Mathematical Programming, 42 (1988), 489-563.

[13] H.N. Gabow: Implementation of Algorithms for Maximum Matching on Nonbipartite Graphs, Ph.D. thesis, Stanford University, 1973.

[14] R. Giles: Optimum matching forests I: Special weights, Mathematical Programming, 22 (1982), $1-11$.

[15] R. Giles: Optimum matching forests II: General weights, Mathematical Programming, 22 (1982), 12-38.

[16] R. Giles: Optimum matching forests III: Facets of matching forest polyhedra, Mathematical Programming, 22 (1982), 39-51. 
[17] J. Keijsper: A Vizing-type theorem for matching forests, Discrete Mathematics, 260 (2003), 211-216.

[18] L. Lovász: Matroid matching and some applications, Journal of Combinatorial Theory, Series $B, 28$ (1980), 208-236.

[19] K. Murota: Characterizing a valuated delta-matroid as a family of delta-matroids, Journal of the Operations Research Society of Japan, 40 (1997), 565-578.

[20] K. Murota: Discrete Convex Analysis, Society for Industrial and Applied Mathematics, Philadelphia, 2003.

[21] A. Schrijver: Total dual integrality of matching forest constraints, Combinatorica, 20 (2000), $575-588$.

[22] A. Schrijver: Combinatorial Optimization-Polyhedra and Efficiency, Springer-Verlag, Heidelberg, 2003.

[23] V.G. Vizing: Ob otsenke khromaticheskogo klassa p-grapha (in Russian), Diskretnyı̆, 3 (1964), 25-30.

[24] V.G. Vizing: Khromaticheskiı̌ klass mul'tigrafa (in Russian), Kibernetika, 1965:3 (1965), 29 39.

[25] W. Wenzel: $\Delta$-matroids with the strong exchange conditions, Applied Mathematics Letters, 6 (1993), 67-70.

[26] W. Wenzel: Pfaffian forms and $\Delta$-matroids, Discrete Mathematics, 115 (1993), 253-266. 